\title{
A Boltzmann-type approach to the formation of wealth distribution curves
}

\author{
Bertram Düring, Daniel Matthes, Giuseppe Toscani ${ }^{\ddagger}$
}

October 7, 2008

\begin{abstract}
Kinetic market models have been proposed recently to account for the redistribution of wealth in simple market economies. These models allow to develop a qualitative theory, which is based on methods borrowed from the kinetic theory of rarefied gases. The aim of these notes is to present a unifying approach to the study of the evolution of wealth in the largetime regime. The considered models are divided into two classes: the first class is such that the society's mean wealth is conserved, while for models of the second class, the mean wealth grows or decreases exponentially in time. In both cases, it is possible to classify the most important feature of the steady (or self-similar, respectively) wealth distributions, namely the fatness of the Pareto tail. We shall also discuss the tails' dynamical stability in terms of the model parameters. Our results are derived by means of a qualitative analysis of the associated homogeneous Boltzmann equations. The key tools are suitable metrics for probability measures, and a concise description of the evolution of moments. A recent extension to economies, in which different groups of agents interact, is presented in detail. We conclude with numerical experiments that confirm the theoretical predictions.
\end{abstract}

Keywords. Econophysics, Boltzmann equation, wealth and income distributions, Pareto distribution, mixtures.

\footnotetext{
*Institut für Analysis und Scientific Computing, Technische Universität Wien, Wiedner Hauptstraße 8-10, 1040 Wien, Austria. e.mail: bduering@anum.tuwien.ac.at

†Institut für Analysis und Scientific Computing, Technische Universität Wien, Wiedner Hauptstraße 8-10, 1040 Wien, Austria. e.mail: matthes@asc.tuwien.ac.at

¥Dipartimento di Matematica, Università degli studi di Pavia, 27100 Pavia, Italy, e.mail: giuseppe.toscani@unipv.it
} 


\section{Contents}

1 Introduction $\quad 3$

2 Economic and kinetic dictionaries $\quad 6$

2.1 Wealth distributions ................ 6

2.2 Wasserstein and Fourier based distances . . . . . . . . . . . . . . 7

2.3 Other Fourier based distances . . . . . . . . . . . . . . . . 10

2.4 One-dimensional Boltzmann models . . . . . . . . . . . . . . . 11

3 Analysis of conservative models $\quad 12$

3.1 Pareto tail of the wealth distribution . . . . . . . . . . . . . 12

3.2 Pointwise conservative models . . . . . . . . . . . . . . 13

3.3 Conservative in the mean models . . . . . . . . . . . . . . . . . 14

3.4 Mathematical details . . . . . . . . . . . . . . . . 16

3.4.1 Evolution of Fourier metrics . . . . . . . . . . . . 16

3.4.2 Evolution of moments . . . . . . . . . . . . . . 17

3.4.3 Existence and tails of the steady state . . . . . . . . . 21

3.4.4 Regularity of the steady wealth distribution . . . . . . . 23

4 Nonconservative models $\quad 26$

4.1 Slanina's model . . . . . . . . . . . . . . . . . . 27

4.1.1 Self-similar solutions . . . . . . . . . . . . . . 27

4.1.2 A Fokker-Planck approximation . . . . . . . . . . . . 28

4.1.3 Fokker-Planck equations . . . . . . . . . . . . . . . 29

4.2 Large-time behavior of nonconservative economies . . . . . . . . 30

4.2.1 Convergence to self-similar solutions . . . . . . . . . . . 30

4.2.2 Regularity of self-similar solutions . . . . . . . . . . . 32

5 Kinetic models for groups of traders 33

5.1 The analogy with gas mixtures . . . . . . . . . . . . . . 34

5.2 A related system of Fokker-Planck equations . . . . . . . . . . 35

6 Two-dimensional models $\quad 38$

6.1 Saving propensity as additional variable . . . . . . . . . . . 38

6.1.1 Pareto tail of the wealth distribution . . . . . . . . . . . 39

6.1.2 Rates of relaxation: Pareto tail . . . . . . . . . . . . . 41

6.1.3 Rates of relaxation: Dirac delta . . . . . . . . . . . . 42

6.2 A Fokker-Planck equation for distributed trading rate . . . . . . 43

7 Numerical experiments $\quad 44$

7.1 CPT model . . . . . . . . . . . . . . . . . . . . 45

7.2 CCM model . . . . . . . . . . . . . . . . . . . 46

7.3 Winner takes all . . . . . . . . . . . . . . . . 48

7.4 Bimodal distributions . . . . . . . . . . . . . . . . 48

8 Conclusions $\quad 51$ 


\section{Introduction}

In recent years, a number of models has been proposed to account for the evolution of the distribution of wealth in a simple market economy. Among other approaches, kinetic market models are presently of particular interest, see e.g. the various contributions in the recent books $[23,18,55,56]$, or the introductory articles $[43,59]$. The founding idea, dating back to the works of Mandelbrot [45], is that a trading market composed of a sufficiently large number of agents can be described using the laws of statistical mechanics, just like for a physical system composed of many interacting particles. In fact, there is an almost literal translation of concepts: molecules are identified with the agents, the particles' energies correspond to the agents' wealths, and binary collisions translate into trade interactions. This modelling is clearly rather ad hoc, but if one is willing to accept the proposed analogies between trading agents and colliding particles, then various well established methods from statistical physics are ready for application to the field of economy. Most notably, the numerous tools originally devised for the study of the energy distribution in a rarefied gas can now be used to analyze wealth distributions. In this way, the kinetic market models provide one possible explanation for the development of universal profiles in wealth distributions of real economies.

One of the authors (GT) started to be interested in this subject after reading a paper by F. Slanina [54]; there, a clear parallelism between the evolution of wealth in a simple economy and the evolution of the particle density in a one-dimensional dissipative gas has been established. This paper motivated to eventually adapt more and more of the ideas, which have been developed in the studies of dissipative Maxwell gases, to the economic framework. (For an introduction to the concepts of Maxwell gases, we refer to [11], and to $[4,6,7,8]$ for further information.)

It should be emphasized, however, that there are substantial differences between the collision mechanism for molecules and the modelling of trade interactions. In the new framework, interactions typically lack the usual microscopic conservation laws for (the analogues of) impulse and energy; moreover, random effects play a crucial rôle. In fact, the key step in establishing a reasonable kinetic market model is the definition of sensible rules on the microscopic level, i.e., the prescription of how wealth is exchanged in trades. Such rules are usually derived from plausible assumptions in an ad hoc manner. (This is clearly in contrast to the original Boltzmann equation, where the microscopic collisions are governed by the laws of classical mechanics.)

The corresponding output of the model are the macroscopic statistics of the wealth distribution in the society. The comparison of this output with realistic data is up to now the only means to evaluate - a posteriori - the quality of a proposed model. For instance, it is commonly accepted that the wealth distribution should approach a stationary (or, in general, a self-similar) profile for large times, and that the latter should exhibit a Pareto tail. Such overpopulated tails are a manifestation of the existence of an upper class of very rich agents, i.e. an indication of an unequal distribution of wealth. The various 
articles in [23] provide an overview over historical and recent studies on the shape of wealth distributions; see also [20] for a collection of relevant references.

In general, the richness of the steady states for kinetic market models is another remarkable difference to the theory of Maxwell molecules. While the Maxwell distribution is the universal steady profile for the velocity distribution of molecular gases, the stationary profiles for wealth can be manifold, and are in general not explicitly known analytically. In fact, they depend heavily on the precise form of the microscopic modelling of trade interactions. Consequently, in investigations of the large-time behavior of the wealth distribution, one is typically limited to describe a few analytically accessible properties (e.g. moments and smoothness) of the latter. A noteworthy exception of a model for which the self-similar profile is know has been found in [54] for an exponentially growing economy in which agents are rewarded for trade interactions proportional to their current wealth. The solution corresponds to the self-similar solution of a one-dimensional dissipative Boltzmann equation of Maxwell type [4].

A variety of models has been proposed and numerically studied in view of the relation between parameters in the microscopic rules and the resulting macroscopic statistics. The features typically incorporated in kinetic trade models are saving effects and randomness. Saving means that each agent is guaranteed to retain at least a certain minimal fraction of his initial wealth at the end of the trade. This concept has probably first been introduced in [17], where a fixed saving rate for all agents has been proposed. Randomness means that the amount of wealth changing hands is non-deterministic. Among others, this idea has been developed in [29], in order to include the effects of a risky market. Depending upon the specific choice of the saving mechanism and the stochastic nature of the trades, the studied systems produce wealth curves with the desired Pareto tail - or not.

In these notes, we analyze and compare a selection of recently developed models. Mainly, we will split our analysis on two different types of interactions. The first type is such that the binary trade is conservative, either microscopically, or in the statistical mean. In this situation, the mean wealth in the model Boltzmann equation is preserved, and one expects the formation of a stationary profile. In the second type of interaction, the mean wealth is not preserved, and therefore the long-time behavior of the wealth distribution is not described by the approach of a stationary, but rather of a self-similar profile.

In the class of conservative trades, the focus is on models with risky investments, originally introduced by Cordier, Pareschi and one of the authors [29], and on variants of the model designed by Chakraborti and Chakrabarti [17]. The applied analytical techniques, however, easily generalize to a broader class of conservative economic games. These techniques have been applied in the current mathematical literature $[29,50,46,47,36,34]$, where kinetic econophysics has been treated in the framework of Maxwell-type molecules. These mathematical results are briefly reviewed, before they are applied to the specific models under consideration. The interest reader, who wishes to obtain a deeper understanding of the mathematical roots (and possible extensions) of the applied tools, is referred e.g. to $[57,16]$. 
For the treatment of the class of non-conservative trades, our starting point is the simplified model introduced by Slanina [54], which has subsequently been studied by Pareschi and one of the authors [50]. This model can be easily generalized to random mixing parameters, in order to include risky market effects. The risky effects are designed with a certain bias to increase the total wealth in trade interactions; this is in contrast to the conservative approach in [29], where risky gains and losses balance in the statistical mean.

As a further approach, related to conservative models, we discuss a kinetic model for wealth distribution in a market which comprises a whole number of countries, or alternatively different social groups within the same country [37]. The goal is to verify analytically the existence of a bimodal stationary distribution [42]. Bimodal distributions (and a polymodal distribution, in general) are, in fact, reported with real data for the income distributions in Argentina [38]. In the proposed model, a bimodal steady state can indeed be obtained, e.g. when the saving parameter takes only two fixed values, which are sufficiently widely separated. The population thus consists of two distinctly different groups of people: some of them tend to save a very large (fixed) fraction of their wealth, while the others tend to save a relatively small fraction. The analytical observation is confirmed in computer experiments: the numerical output evolves towards a robust and distinct two-peak distribution as the difference in the two saving parameters is increased systematically.

The kinetic approach presented in these notes is complementary to the numerous theoretical and numerical studies that can be found in the recent physics literature on the subject, from which it differs in several subtle points. In particular, the analysis is entirely based on the spatially homogeneous Boltzmann equation associated to the microscopic trade rules of the respective model. Thus, here agents on the market are treated as a continuum, just like molecules in classical gas dynamics. Not only does this approach constitute the most natural generalization of the classical ideas to econophysics. But moreover, it clarifies that certain peculiar observations made in ensembles of finitely many agents and in numerical experiments (like the apparent creation of steady distributions of infinite average wealth, e.g. $[21,22,20])$ are genuine finite size effects. Unfortunately, this approach, while powerful and robust, is up to now almost ignored by the pertinent literature of the econophysics community. To close this gap, propaganda to the physicists community has been made by resorting to a short description both of the mathematical models and methods in a well addressed physical journal [35]. We will borrow from this paper, from which the present notes differ mainly in the mathematical details.

For the sake of uniformity, we restrict ourselves in these notes to markets characterized by binary trades. Other kinetic models have been recently proposed, which, while maintaining the kinetic description, introduce more sophisticated rules for trading. For example, a description of the behavior of a stock price has been developed by Cordier, Pareschi and Piatecki in [28]. Further, we mention that there are efforts to include non-microscopic effects, like global taxation (and subsequent redistribution), for example in a recent work of Garibaldi, Scalas and Viarengo [41]. 
To conclude this introduction, a comment on the justification of kinetic market models is in place. The socio-economic behavior of a (real) population of agents is certainly extremely complex. Apart from elements from mathematics and economics, a sound description — if one at all exists — would necessarily need contributions from various other fields, like psychology. Clearly, the mathematical models presented in these notes are too simple to even pretend to reflect the real situation. However, the idea to describe economic trades in terms of a kinetic equation gives rise to a variety of challenging mathematical problems, both from the theoretical and numerical point of view. In particular, it is remarkable that this class of simple models possesses such a wide spectrum of possible equilibria (some of which indeed resemble realistic wealth distributions). Moreover, kinetic market models are extremely flexible with respect to the introduction of additional effects. In this way, the described models should be considered as basic building blocks, that can easily be combined, adapted and improved. Hopefully, the reading of these notes will be encouraging for the introduction of more realistic models in the same spirit.

\section{$2 \quad$ Economic and kinetic dictionaries}

\subsection{Wealth distributions}

In a closed ensemble of agents (i.e. a market), the wealth distribution $f(t ; w)$ refers to the relative density of agents with wealth $w$ at time $t \geq 0$. Debts are excluded in the models considered here, i.e. $f(t ; w)=0$ for $w<0$, but concentration in $w=0$ is allowed. The first moment of $f(t ; w)$ yields the average wealth per agent,

$$
M_{1}(t)=\int_{\mathbb{R}_{+}} w f(t ; w) d w .
$$

In the models under consideration, the density $f(t ; w)$ stabilizes at some stationary wealth curve $f_{\infty}(w)$ in the large-time limit $t \rightarrow \infty$. The central notion in the theory of wealth distributions is that of the celebrated Pareto index $\alpha \geq 1$. This number describes the size of the rich upper class in the considered ensemble of agents. Roughly, the smaller $\alpha$ is, the more of the total wealth is concentrated in the hands of a small group of individuals.

The stationary curve $f_{\infty}(w)$ satisfies the Pareto law [51] with index $\alpha$, provided that $f_{\infty}$ decays like an inverse power function for large $w$,

$$
f_{\infty}(w) \propto w^{-(\alpha+1)} \quad \text { as } w \rightarrow+\infty .
$$

More precisely, $f_{\infty}$ has Pareto index $\alpha \in[1,+\infty)$ if the moments

$$
M_{s}:=\int_{\mathbb{R}_{+}} w^{s} f_{\infty}(w) d w
$$

are finite for all positive $s<\alpha$, and infinite for $s>\alpha$. If all $M_{s}$ are finite (e.g. for a Gamma distribution), then $f_{\infty}$ is said to possess a slim tail. 
According to empirical data from ancient Egypt until today [23, 20], the wealth distribution among the population in a capitalistic country follows the Pareto law, with an index $\alpha$ ranging between 1.5 and 2.5. Slim tails are typical for societies with a highly equal distribution of wealth. Intuitively, one may think of socialist countries.

Surprisingly, the mathematical description of the stationary wealth curve $f_{\infty}$ attracted the interest of mathematicians many years before Mandelbrot works [45]. A description of this curve by means of a generalized Gamma distribution is due to Amoroso [1] and D'Addario [30]. If one assumes for $f_{\infty}$ a unit mean, the Amoroso distribution reads

$$
f_{\alpha}(w)=\frac{(\alpha-1)^{\alpha}}{\Gamma(\alpha)} \frac{\exp \left(-\frac{\alpha-1}{w}\right)}{w^{1+\alpha}}, \quad \alpha>1 .
$$

Note that this stationary distribution exhibits a Pareto power law tail of order $\alpha$ for large $w$ 's.

\subsection{Wasserstein and Fourier based distances}

Since Monte Carlo simulations produce distributions of point masses instead of smooth curves, a good notion of distance between measures is important to quantify the convergence of numerical results to the continuous limit. In most of our applications, we will consider probability distributions possessing finite moments of some order $s>1$. Accordingly, for given constants $c>0$ and $s>1$, define $\mathcal{M}_{c, s}$ as the set of (Borel) probability measures on $\mathbb{R}_{+}$satisfying

$$
\int_{\mathbb{R}_{+}} w f(w) d w=c, \quad \int_{\mathbb{R}_{+}} w^{r} f(w) d w<\infty .
$$

Among other distances, the Wasserstein distance (of order one) of two density functions $f_{1}(w), f_{2}(w)$ is an extremely useful instrument. This distance is given by

$$
\mathbf{W}\left[f_{1}, f_{2}\right]:=\int_{\mathbb{R}_{+}}\left|F_{1}(v)-F_{2}(v)\right| d v,
$$

where the $F_{i}$ denote the distribution functions,

$$
F_{i}(v)=\int_{v}^{\infty} f_{i}(w) d w \quad(i=1,2) .
$$

Equivalently, the Wasserstein distance is defined as the infimum of the costs for transportation [57],

$$
\mathbf{W}\left[f_{1}, f_{2}\right]:=\inf _{\pi \in \Pi} \int|v-w| d \pi(v, w) .
$$

Here $\Pi$ is the collection of all measures in the plane $\mathbb{R}^{2}$ with marginal densities $f_{1}$ and $f_{2}$, respectively. The infimum is in fact a minimum, and is realized 
by some optimal transport plan $\pi_{\text {opt }}$. Convergence of densities $f(t ; w)$ to a limit $f_{\infty}(w)$ in the Wasserstein distance is equivalent to the weak convergence $f(t ; w) d w \rightarrow f_{\infty}(w) d w$ in the sense of measures, and convergence of the first moments. Note that definition $(2.8)$ is a particular case $(p=1)$ of the general expression of the Wasserstein distance of order $p>0$,

$$
\mathbf{W}_{p}\left[f_{1}, f_{2}\right]^{p}:=\inf _{\pi \in \Pi} \int|v-w|^{p} d \pi(v, w) .
$$

There is an intimate relation of Wasserstein to Fourier metrics [40], defined by

$$
d_{s}\left[f_{1}, f_{2}\right]=\sup _{\xi}\left[|\xi|^{-s}\left|\hat{f}_{1}(\xi)-\hat{f}_{2}(\xi)\right|\right], \quad s>0,
$$

where $\hat{f}(t ; \xi)$ is the Fourier transform of $f(t ; x)$,

$$
\widehat{f}(t ; \xi)=\int_{\mathbb{R}_{+}} e^{-i \xi v} f(t ; v) d v
$$

Note that the distance (2.10) is finite for some $s>1$ if the distribution functions have the same moments up to $[s]$, where $[s]$ denotes as usual the entire part of $s$. The interested reader can have an almost complete picture of the key properties of these metrics by looking at the notes [16]. There, however, mostly the case of the Wasserstein distance of order two (assuming finite second moment of the occurring densities) is considered. In the economic framework, where the typical case is $p=1$, for $s>1$, the Wasserstein and Fourier distance are related [34] by

$$
\mathbf{W}\left[f_{1}, f_{2}\right] \leq C\left(d_{s}\left[f_{1}, f_{2}\right]\right)^{-(s-1) / s(2 s-1)} .
$$

We sketch below the proof of this result.

Lemma 2.1. Assume that two probability densities $f$ and $g$ have first moment equal to one, and some moment of order $s \in(1,2]$ bounded. Then there exists a constant $C>0$, depending only on $s$ and the values of the $s$-th moments of $f$ and $g$, such that

$$
\mathbf{W}[f, g] \leq C\left(d_{s}[f, g]\right)^{\frac{s-1}{s(2 s-1)}} .
$$

Conversely, one has

$$
d_{1}[f, g] \leq \mathbf{W}[f, g]
$$

even if no moments of $f$ and $g$ above the first are bounded.

Proof. To prove (2.12), we adapt the proof of Theorem 2.21 in [16], corresponding to $s=2$. Define

$$
M=\max \left\{\int_{\mathbb{R}_{+}} v^{s} f(v) d v, \int_{\mathbb{R}_{+}} v^{s} g(v) d v\right\} .
$$


Starting from the definition of the Wasserstein distance in (2.6), we estimate

$$
\begin{aligned}
\mathbf{W}[f, g] & =\int_{\mathbb{R}_{+}}|F(v)-G(v)| d v \\
& \leq \int_{0}^{R}|F(v)-G(v)| d v+R^{1-s} \int_{R}^{\infty} v^{s-1}|F(v)-G(v)| d v \\
& \leq R^{1 / 2}\left(\int_{\mathbb{R}_{+}}|F(v)-G(v)|^{2} d v\right)^{1 / 2}+R^{1-s} \int_{R}^{\infty} v^{s-1}|F(v)-G(v)| d v
\end{aligned}
$$

where the parameter $R=R(t)>0$ is specified later. By Parseval's identity,

$$
\begin{aligned}
\int_{\mathbb{R}_{+}}|(F-G)(v)|^{2} d v & =\int_{\mathbb{R}^{-}}|(\widehat{F-G})(\xi)|^{2} d \xi=\int_{\mathbb{R}}\left|(i \xi)^{-1}(\hat{f}(\xi)-\hat{g}(\xi))\right|^{2} d \xi \\
& \leq\left(d_{s}[f, g]\right)^{2} \int_{|\xi|<r}|\xi|^{2(s-1)} d \xi+4 \int_{|\xi| \geq r} \xi^{-2} d \xi \\
& =(2 s-1)^{-1} r^{2 s-1}\left(d_{s}[f, g]\right)^{2}+8 r^{-1} \\
& \leq C_{1}\left(d_{s}[f, g]\right)^{1 / s}
\end{aligned}
$$

The last estimate follows by optimizing in the previous line with respect to $r>0$. The constant $C_{1}$ depends only on $s>1$. This gives a bound on the first term in (2.14) above. We estimate the second term, integrating by parts:

$$
\begin{aligned}
\int_{R}^{\infty} v^{s-1}|F(v)-G(v)| d v & \leq \int_{R}^{\infty} v^{s-1}(F(v)+G(v)) d v \\
& =\frac{1}{s} \int_{R}^{\infty} v^{s}(f(v)+g(v)) d v+\left.\left(v^{s}(F(v)+G(v))\right)\right|_{R} ^{\infty} \\
& \leq \frac{2 M}{s}+\lim _{r \rightarrow+\infty}\left(r^{s}(F(r)+G(r))\right)
\end{aligned}
$$

The last expression is easily estimated by Chebyshev's inequality, i.e.,

$$
\lim _{r \rightarrow \infty}\left(r^{s} F(r)\right) \leq \lim _{r \rightarrow \infty}\left(r^{s} \mathrm{P}_{f}[v>r]\right) \leq \lim _{r \rightarrow \infty} \int_{r}^{\infty} v^{s} f(v) d v=0,
$$

since the $s$-th moment of $f$ is finite. In summary, (2.14) yields

$$
\mathbf{W}[f, g] \leq C_{1}^{1 / 2} R^{1 / 2}\left(d_{s}[f, g]\right)^{1 /(2 s)}+2 s^{-1} M R^{1-s} .
$$

Optimizing this over $R$ yields the desired inequality (2.12).

The other inequality (2.13) is derived from the alternative definition (2.6) of 
$\mathbf{W}[f, g]$, with $\pi_{\text {opt }}$ being the optimal transport plan

$$
\begin{aligned}
d_{1}[f, g] & =\sup _{\xi \neq 0}\left(|\xi|^{-1}\left|\int_{\mathbb{R}_{+}} e^{-i v \xi} f(v) d v-\int_{\mathbb{R}_{+}} e^{-i w \xi} g(w) d w\right|\right) \\
& \leq \sup _{\xi \neq 0}\left(|\xi|^{-1} \int_{\mathbb{R}_{+}^{2}}\left|e^{-i v \xi}-e^{-i w \xi}\right| d \pi_{o p t}(v, w)\right) \\
& \leq \int_{\mathbb{R}_{+}^{2}} \sup _{\xi \neq 0}\left(\frac{\left|1-e^{i(v-w) \xi}\right|}{|v-w||\xi|}\right)|v-w| d \pi_{o p t}(v, w) \\
& =\sup _{x \in \mathbb{R}}\left(\frac{\left|1-e^{i x}\right|}{|x|}\right) \mathbf{W}[f, g] .
\end{aligned}
$$

In view of the elementary inequality $|1-\exp (i x)| \leq|x|$ for $x \in \mathbb{R}$, this yields the claim (2.13).

Examples. Two Dirac distributions have Wasserstein distance $\mathbf{W}\left[\delta_{x}, \delta_{y}\right]=$ $|x-y|$. Likewise, $d_{1}\left[\delta_{x}, \delta_{y}\right]=|x-y|$, but notice that $d_{s}\left[\delta_{x}, \delta_{y}\right]=+\infty$ for $s>1$ unless $x=y$. More generally, a density $f_{1}(v)$ and its translate $f_{2}(v)=f_{1}(v-z)$ have Wasserstein distance $\mathbf{W}\left[f_{1}, f_{2}\right]=|z|$ and Fourier distance $d_{1}\left[f_{1}, f_{2}\right]=|z|$. For comparison, if $f_{1}$ is supported in a small interval $[-\epsilon,+\epsilon]$, then $\left\|f_{1}-f_{2}\right\|_{L^{1}}=$ 2 for all $|z|>\epsilon$. Thus, both the Wasserstein and the Fourier based distances provide a more sensible notion of "closeness" of densities than e.g. the classical $L^{1}$-distance.

\subsection{Other Fourier based distances}

One of the weak points of the Fourier based distance (2.10) is that, for a given $s$ such that $1<s<2$, it is not known if the space of probability measures $\mathcal{M}_{c, r}$ with metric $d_{s}$ is complete or not. This unpleasant fact is discussed in [16], together with a possible remedy. A further metric, however, can be introduced, which does not have the same problem, while it possesses most of the properties of the metric $d_{s}$. This metric has been introduced in [3] to characterize fixed points of convex sums of random variables with a small number of moments. For $s \in(1,2)$,

$$
D_{s}\left[f_{1}, f_{2}\right]=\int|\xi|^{-(s+1)}\left|\hat{f}_{1}(\xi)-\hat{f}_{2}(\xi)\right| d \xi, \quad s>0 .
$$

As proven in [3], $\left(\mathcal{M}_{c, s}, D_{s}\right)$ is complete. A proof of the analogous of Lemma 2.1 would be desirable.

Let $f_{\mu}(w)=\frac{1}{\mu} f\left(\frac{w}{\mu}\right)$. Then, the metric (2.15) is such that

$$
D_{s}\left[f_{\mu}, g_{\mu}\right]=\mu^{s} D_{s}[f, g] .
$$

The scaling property (2.16), which holds also for the metric $d_{s}$, is at the basis of most of the applications of Fourier based metrics to kinetic models. 


\subsection{One-dimensional Boltzmann models}

Here we consider a class of models in which agents are indistinguishable. Then, an agent's "state" at any instant of time $t \geq 0$ is completely characterized by his current wealth $w \geq 0$. When two agents encounter in a trade, their pre-trade wealths $v, w$ change into the post-trade wealths $v^{*}, w^{*}$ according to the rule

$$
v^{*}=p_{1} v+q_{1} w, \quad w^{*}=q_{2} v+p_{2} w .
$$

The interaction coefficients $p_{i}$ and $q_{i}$ are non-negative random variables. While $q_{1}$ denotes the fraction of the second agent's wealth transferred to the first agent, the difference $p_{1}-q_{2}$ is the relative gain (or loss) of wealth of the first agent due to market risks. We assume that $p_{i}$ and $q_{i}$ have fixed laws, which are independent of $v$ and $w$, and of time.

In one-dimensional models, the wealth distribution $f(t ; w)$ of the ensemble coincides with agent density and satisfies the associated spatially homogeneous Boltzmann equation,

$$
\partial_{t} f+f=Q_{+}(f, f),
$$

on the real half line, $w \geq 0$. The collisional gain operator $Q_{+}$acts on test functions $\varphi(w)$ as

$$
\begin{aligned}
Q_{+}(f, f)[\varphi] & :=\int_{\mathbb{R}_{+}} \varphi(w) Q_{+}(f, f)(w) d w \\
& =\frac{1}{2} \int_{\mathbb{R}_{+}^{2}}\left\langle\varphi\left(v^{*}\right)+\varphi\left(w^{*}\right)\right\rangle f(v) f(w) d v d w
\end{aligned}
$$

with $\langle\cdot\rangle$ denoting the expectation with respect to the random coefficients $p_{i}$ and $q_{i}$ in (2.17). The large-time behavior of the density is heavily dependent of the evolution of the average wealth

$$
M(t):=M_{1}(t)=\int_{\mathbb{R}_{+}} w f(t ; w) d w,
$$

Conservative models are such that the average wealth of the society is conserved with time, $M(t)=M$, and we will generally assume that the value of $M$ to be finite. In terms of the interaction coefficients, this is equivalent to $\left\langle p_{1}+q_{2}\right\rangle=$ $\left\langle p_{2}+q_{1}\right\rangle=1$.

Non conservative models are such that $M(t)$ is not conserved with time. We will restrict ourselves to the case in which $\left\langle p_{1}+q_{2}\right\rangle=\left\langle p_{2}+q_{1}\right\rangle \neq 1$, so that the average wealth is exponentially increasing or decreasing

$$
M(t)=M(0) e^{\left(\left\langle p_{1}+q_{2}\right\rangle-1\right) t} .
$$

From the point of view of its kinetic classification, the Boltzmann equation (2.18) belongs to the Maxwell type. In the Boltzmann equation for Maxwell molecules, in fact, the collision frequency is independent of the relative velocity [10], and the loss term in the collision operator is linear. This introduces a great simplification, that allows to use most of the well established techniques developed for the three-dimensional spatially homogeneous Boltzmann equation for Maxwell molecules in the field of wealth redistribution. 


\section{Analysis of conservative models}

First, we shall give an overview on the available analytical results for conservative models, and indicate the derivation of these results on an intuitive, nonrigorous level. The differences between pointwise conservative and conservativein-the-mean models are discussed. Subsequently, some mathematical details and proofs are provided in section 3.4.

\subsection{Pareto tail of the wealth distribution}

We introduce the characteristic function

$$
\mathbf{S}(s)=\frac{1}{2}\left(\sum_{i=1}^{2}\left\langle p_{i}^{s}+q_{i}^{s}\right\rangle\right)-1,
$$

which is convex in $s>0$, with $\mathbf{S}(0)=1$. Also, $\mathbf{S}(1)=0$ because of the conservation property (2.20). The results from [46, 34] imply the following. Unless $\mathbf{S}(s) \geq 0$ for all $s>0$, any solution $f(t ; w)$ tends to a steady wealth distribution $P_{\infty}(w)=f_{\infty}(w)$, which depends on the initial wealth distribution only through the conserved mean wealth $M>0$. Moreover, exactly one of the following is true:

$(\mathrm{PT})$ if $\mathbf{S}(\alpha)=0$ for some $\alpha>1$, then $P_{\infty}(w)$ has a Pareto tail of index $\alpha$;

$(\mathrm{ST})$ if $\mathbf{S}(s)<0$ for all $s>1$, then $P_{\infty}(w)$ has a slim tail;

(DD) if $\mathbf{S}(\alpha)=0$ for some $0<\alpha<1$, then $P_{\infty}(w)=\delta_{0}(w)$, a Dirac Delta at $w=0$.

To derive these results, one studies the evolution equation for the moments

$$
M_{s}(t):=\int_{\mathbb{R}_{+}} w^{s} f(t ; w) d w
$$

which is obtained by integration of (2.18) against $\varphi(w)=w^{s}$,

$$
\frac{d}{d t} M_{s}=Q_{+}[\varphi]-M_{s}
$$

Using an elementary inequality for $x, y \geq 0, s \geq 1$,

$$
x^{s}+y^{s} \leq(x+y)^{s} \leq x^{s}+y^{s}+2^{s-1}\left(x y^{s-1}+x^{s-1} y\right),
$$

in (2.19), one calculates for the right-hand side of (3.24)

$$
\mathbf{S}(s) M_{s} \leq Q_{+}[\varphi]-M_{s} \leq \mathbf{S}(s) M_{s}+2^{s-2} \sum_{i=1}^{2}\left\langle p_{i} q_{i}^{s-1}+p_{i}^{s-1} q_{i}\right\rangle M M_{s}^{1-1 / s} .
$$

Solving (3.24) with (3.26), one finds that either $M_{s}(t)$ remains bounded for all times when $\mathbf{S}(s)<0$, or it diverges like $\exp [t \mathbf{S}(s)]$ when $\mathbf{S}(s)>0$, respectively. 
In case (PT), exactly the moments $M_{s}(t)$ with $s>\alpha$ blow up as $t \rightarrow \infty$, giving rise to a Pareto tail of index $\alpha$. We emphasize that $f(t ; w)$ possesses finite moments of all orders at any finite time. The Pareto tail forms in the limit $t \rightarrow \infty$.

In case (ST), all moments converge to limits $M_{s}(t) \rightarrow M_{s}^{*}$, so the tail is slim. One can obtain additional information on the stationary wealth distribution $P_{\infty}(w)$ from the recursion relation for the principal moments,

$$
-\mathbf{S}(s) M_{s}^{*}=\frac{1}{2} \sum_{k=1}^{s-1} \sum_{i=1}^{2}\left(\begin{array}{l}
s \\
k
\end{array}\right)\left\langle p_{i}^{k} q_{i}^{s-k}\right\rangle M_{k}^{*} M_{s-k}^{*}, \quad s=2,3, \ldots
$$

The latter is obtained by integration of (2.18) against $\varphi(w)=w^{s}$ in the steady state $\partial_{t} f=0$.

In case (DD), all moments $M_{s}(t)$ with $s>1$ blow up. The underlying process is a separation of wealth as time increases: while more and more agents become extremely poor, fewer and fewer agents possess essentially the entire wealth of the society. In terms of $f(t ; w)$, one observes an accumulation in the pauper region $0 \leq w \ll 1$, while the density rapidly spreads into the region $w \gg 1$. The expanding support of $f(t ; w)$ is balanced by a decrease in magnitude, since the average wealth is fixed. This induces a pointwise convergence $f(t ; w) \rightarrow 0$ for all $w>0$. Such a condensation of wealth has been observed and described in several contexts $[44,14,15,24]$ before.

An illustration of the solution's behavior in the (DD) case is provided by the "Winner takes all" dynamics, with rules

$$
v^{*}=v+w, \quad w^{*}=0 .
$$

In each trade, the second agent loses all of his wealth to the first agent. The solution for the initial condition $f(0 ; w)=\exp (-w)$ is explicit,

$$
f(t ; w)=\left(\frac{2}{2+t}\right)^{2} \exp \left(-\frac{2}{2+t} w\right)+\frac{t}{2+t} \delta_{0}(w) .
$$

Note that the average wealth is conserved at all finite times $t \geq 0$, so that $\lim _{t \rightarrow \infty} M_{1}(t)=M_{1}(0)$, but $f_{\infty}=\delta_{0}$ has vanishing average wealth.

\subsection{Pointwise conservative models}

The first explicit description of a binary wealth exchange model dates back to Angle [2] (although the intimate relation to statistical mechanics was only described about one decade later [44, 33]): in each binary interaction, winner and loser are randomly chosen, and the loser yields a random fraction of his wealth to the winner. From here, Chakraborti and Chakrabarti [17] developed the class of strictly conservative exchange models, which preserve the total wealth in each individual trade,

$$
v^{*}+w^{*}=v+w
$$


In its most basic version, the microscopic interaction is determined by one single parameter $\lambda \in(0,1)$, which is the global saving propensity. In interactions, each agent keeps the corresponding fraction of his pre-trade wealth, while the rest $(1-\lambda)(v+w)$ is equally shared among the two trade partners,

$$
v^{*}=\lambda v+\frac{1}{2}(1-\lambda)(v+w), \quad w^{*}=\lambda w+\frac{1}{2}(1-\lambda)(v+w) .
$$

In result, all agents become equally rich eventually. Indeed, the stochastic variance of $f(t ; w)$ satisfies

$$
\frac{d}{d t} \int_{\mathbb{R}_{+}}(w-M)^{2} f(t ; w) d w=-\frac{1}{2}\left(1-\lambda^{2}\right) \int_{\mathbb{R}_{+}}(w-M)^{2} f(t ; w) d w .
$$

The steady state $f_{\infty}(w)=\delta_{M}(w)$ is a Dirac Delta concentrated at the mean wealth, and is approached at the exponential rate $\left(1-\lambda^{2}\right) / 2$.

More interesting, non-deterministic variants of the model have been proposed, where the amount $(1-\lambda)(v+w)$ is not equally shared, but in a stochastic way:

$$
v^{*}=\lambda v+\epsilon(1-\lambda)(v+w), \quad w^{*}=\lambda w+(1-\epsilon)(1-\lambda)(v+w),
$$

with a random variable $\epsilon \in(0,1)$. Independently of the particular choice of $\epsilon$, the characteristic function

$$
\mathbf{S}(s)=\frac{1}{2}\left(\left\langle[\lambda+\epsilon(1-\lambda)]^{s}\right\rangle+\left\langle[1-\epsilon(1-\lambda)]^{s}\right\rangle+\left[\left\langle\epsilon^{s}\right\rangle+\left\langle(1-\epsilon)^{s}\right\rangle\right](1-\lambda)^{s}\right)-1
$$

is negative for all $s>1$, hence case (ST) applies. Though the steady state $f_{\infty}$ is no longer explicit — for approximations see [26, 53] — one concludes that its tail is slim. In conclusion, no matter how sophisticated the trade mechanism is chosen, one-dimensional, strictly conservative trades always lead to narrow, "socialistic" distributions of wealth.

\subsection{Conservative in the mean models}

Cordier et al. [29] have introduced the CPT model, which breaks with the paradigm of strict conservation. The idea is that wealth changes hands for a specific reason: one agent intends to invest his wealth in some asset, property etc. in possession of his trade partner. Typically, such investments bear some risk, and either provide the buyer with some additional wealth, or lead to the loss of wealth in a non-deterministic way. An easy realization of this idea [46] consists in coupling the previously discussed rules (3.31) with some risky investment that yields an immediate gain or loss proportional to the current wealth of the investing agent,

$$
v^{*}=\left(\frac{1+\lambda}{2}+\eta_{1}\right) v+\frac{1-\lambda}{2} w, \quad w^{*}=\left(\frac{1+\lambda}{2}+\eta_{2}\right) w+\frac{1-\lambda}{2} v,
$$




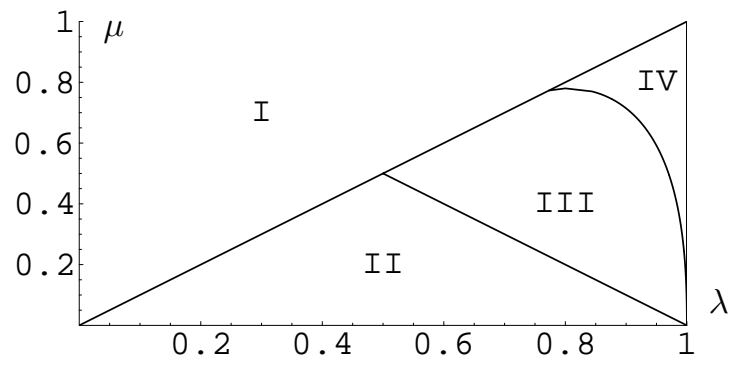

Figure 3.1: Regimes for the formation of Pareto tails.

The coefficients $\eta_{1}, \eta_{2}$ are random parameters, which are independent of $v$ and $w$, and distributed so that always $v^{*}, w^{*} \geq 0$, i.e. $\eta_{1}, \eta_{2} \geq-\lambda$. Unless these random variables are centered, i.e. $\left\langle\eta_{1}\right\rangle=\left\langle\eta_{2}\right\rangle=0$, it is immediately seen that the mean wealth is not preserved, but it increases or decreases exponentially (see the computations in [29]. For centered $\eta_{i}$,

$$
\left\langle v^{*}+w^{*}\right\rangle=\left(1+\left\langle\eta_{1}\right\rangle\right) v+\left(1+\left\langle\eta_{2}\right\rangle\right) w=v+w,
$$

implying conservation of the average wealth. Various specific choices for the $\eta_{i}$ have been discussed [46]. The easiest one leading to interesting results is $\eta_{i}=$ $\pm \mu$, where each sign comes with probability $1 / 2$. The factor $\mu \in(0, \lambda)$ should be understood as the intrinsic risk of the market: it quantifies the fraction of wealth agents are willing to gamble on. Figure 3.1 displays the various regimes for the steady state $f_{\infty}$ in dependence of $\lambda$ and $\mu$, which follow from numerical evaluation of

$$
\mathbf{S}(s)=\frac{1}{2}\left[\left(\frac{1+\lambda}{2}-\mu\right)^{s}+\left(\frac{1+\lambda}{2}+\mu\right)^{s}\right]+\left(\frac{1-\lambda}{2}\right)^{s}-1 .
$$

Zone I is forbidden by the constraint $\mu<\lambda$. In zone II, corresponding to low market risk, the wealth distribution shows again "socialistic" behavior with slim tails. Increasing the risk, one falls into "capitalistic" zone III, where the wealth distribution displays the desired Pareto tail. A minimum of saving $(\lambda>1 / 2)$ is necessary for this passage; this is expected since if wealth is spent too quickly after earning, agents cannot accumulate enough to become rich. Inside zone III, the Pareto index $\alpha$ decreases from $+\infty$ at the border with zone II to unity at the border to zone IV. Finally, in zone IV, the steady wealth distribution is a Delta in zero. Both risk and saving propensity are so high that a marginal number of individuals manages to monopolize all of the society's wealth. In the long-time limit, these few agents become infinitely rich, leaving all other agents truly pauper. 


\subsection{Mathematical details}

We will now give some details about proofs. One of the main tools is the use of the Fourier transform. This idea, which goes back to the seminal work of Bobylev [9, 10], is well-suited to treat collision kernels of Maxwellian type. In particular, the Fourier representation is particularly adapted to the use of various Fourier metrics. An auxiliary tool is the study of the evolution of moments.

\subsubsection{Evolution of Fourier metrics}

According to the collision rule (2.17), the transformed kernel reads

$$
\widehat{Q}(\hat{f}, \hat{f})(\xi)=\frac{1}{2}\left\langle\widehat{f}\left(p_{1} \xi\right) \widehat{f}\left(q_{1} \xi\right)+\widehat{f}\left(p_{2} \xi\right) \widehat{f}\left(q_{2} \xi\right)\right\rangle-\widehat{f}(\xi) \widehat{f}(0) .
$$

Assuming the initial distribution of wealth in $\mathcal{M}_{m, s}$, with $s>1$, the initial conditions turn into

$$
\widehat{f}_{0}(0)=1 \quad \text { and } \quad \widehat{f}_{0}^{\prime}(0)=i M
$$

Hence, the Boltzmann equation (2.18) can be rewritten as

$$
\frac{\partial \widehat{f}(t ; \xi)}{\partial t}+\widehat{f}(t ; \xi)=\frac{1}{2}\left\langle\widehat{f}\left(p_{1} \xi\right) \widehat{f}\left(q_{1} \xi\right)+\widehat{f}\left(p_{2} \xi\right) \widehat{f}\left(q_{2} \xi\right)\right\rangle=\left\langle\widehat{f}\left(p_{i} \xi\right) \widehat{f}\left(q_{i} \xi\right)\right\rangle_{+} .
$$

Details about existence of solutions to equation (3.39) can be found in [46]. Let $f_{1}$ and $f_{2}$ be two solutions of the kinetic equation (3.39), corresponding to initial values $f_{1,0}$ and $f_{2,0}$ in $\mathcal{M}_{m, s}$, with $s>1$, and denote by $\widehat{f}_{1}, \widehat{f}_{2}$ their Fourier transforms. Let $s \geq 1$ be such that $d_{s}\left(f_{1,0}, f_{2,0}\right)$ is finite. Then

$$
\frac{\partial}{\partial t} \frac{\left(\widehat{f}_{1}(\xi)-\widehat{f}_{2}(\xi)\right)}{|\xi|^{s}}+\frac{\widehat{f}_{1}(\xi)-\widehat{f}_{2}(\xi)}{|\xi|^{s}}=\frac{\left\langle\widehat{f}_{1}\left(p_{i} \xi\right) \widehat{f}_{1}\left(q_{i} \xi\right)-\widehat{f}_{2}\left(p_{i} \xi\right) \widehat{f}_{2}\left(q_{i} \xi\right)\right\rangle_{+}}{|\xi|^{s}} .
$$

Now, since $\left|\widehat{f}_{1}(t ; \xi)\right| \leq 1$ and $\left|\widehat{f}_{2}(t ; \xi)\right| \leq 1$ for all $\xi \in \mathbb{R}$, we obtain

$$
\begin{aligned}
& \left|\frac{\left\langle\widehat{f}_{1}\left(p_{i} \xi\right) \widehat{f}_{1}\left(q_{i} \xi\right)-\widehat{f}_{2}\left(p_{i} \xi\right) \widehat{f}_{2}\left(q_{i} \xi\right)\right\rangle_{+}}{|\xi|^{s}}\right| \\
\leq & \left\langle\left|\widehat{f}_{1}\left(p_{i} \xi\right)\right|\left|\frac{\widehat{f}_{1}\left(q_{i} \xi\right)-\widehat{f}_{2}\left(q_{i} \xi\right)}{\left|q_{i} \xi\right|^{s}}\right| q_{i}^{s}\right\rangle_{+}+\left\langle\left|\widehat{f}_{2}\left(q_{i} \xi\right)\right|\left|\frac{\widehat{f}_{1}\left(p_{i} \xi\right)-\widehat{f}_{2}\left(p_{i} \xi\right)}{\left|p_{i} \xi\right|^{s}}\right| p_{i}^{s}\right\rangle_{+} \\
\leq & \sup _{\xi}\left|\frac{\widehat{f}_{1}(\xi)-\widehat{f}_{2}(\xi)}{|\xi|^{s}}\right|\left\langle p_{i}^{s}+q_{i}^{s}\right\rangle_{+} .
\end{aligned}
$$

In terms of the auxiliary quantity

$$
h(t ; \xi)=\frac{\widehat{f}_{1}(\xi)-\widehat{f}_{2}(\xi)}{|\xi|^{s}},
$$


the preceding computation shows that

$$
\left|\frac{\partial h}{\partial t}+h\right| \leq\left\langle p_{i}^{s}+q_{i}^{s}\right\rangle_{+}\|h\|_{\infty} .
$$

Gronwall's lemma yields at once that

$$
\|h(t)\|_{\infty} \leq \exp \left\{\left(\left\langle p_{i}^{s}+q_{i}^{s}\right\rangle_{+}-1\right) t\right\}\left\|h_{0}\right\|_{\infty} .
$$

This introduces into the game the quantity $\mathbf{S}(s)$ we defined in (3.22). Since $\|h(t)\|_{\infty}=d_{s}\left[f_{1}(t), f_{2}(t)\right]$, we obtain from $(3.41)$

$$
d_{s}\left[f_{1}(t), f_{2}(t)\right] \leq \exp \{\mathbf{S}(s) \cdot t\} d_{s}\left[f_{1,0}, f_{2,0}\right]
$$

In particular, if $\mathbf{S}(s)$ is negative, then the $d_{s}$-distance of $f_{1}$ and $f_{2}$ decays exponentially in time. We remark that, thanks to the scaling property $(2.16)$, the same result holds for the metric $D_{s}$. Thus,

$$
D_{s}\left[f_{1}(t), f_{2}(t)\right] \leq \exp \{\mathbf{S}(s) \cdot t\} D_{s}\left[f_{1,0}, f_{2,0}\right] .
$$

Theorem 3.1. [46] Let $f_{1}(t)$ and $f_{2}(t)$ be two solutions of the Boltzmann equation (2.18), corresponding to initial values $f_{1,0}$ and $f_{2,0}$ in $\mathcal{M}_{M, r}, r>1$. Let $s \geq 1$ be such that $d_{s}\left[f_{1,0}, f_{2,0}\right]$ is finite. Then, for all times $t \geq 0$, (3.42) and (3.43) hold.

In particular, if $\mathbf{S}(s)$ is negative, then the Fourier based distances of $f_{1}$ and $f_{2}$ decay exponentially in time.

Putting $f_{1,0}=f_{2,0}=f_{0}$ in (3.42), and using $s=1$ yields

Corollary 3.2. If $f_{0}$ is a nonnegative density in $\mathcal{M}_{M, r}, r>1$. then there exists a unique weak solution $f(t)$ of the Boltzmann equation with $f(0)=f_{0}$.

\subsubsection{Evolution of moments}

In Theorem 3.1 about the large-time behavior of solutions to (2.18), the essential quantity $\mathbf{S}$ has been introduced. Below, we prove that the values $\mathbf{S}(s)$ also control the asymptotic behavior of moments. In fact, if $\mathbf{S}(s)$ is negative for some $s>0$, then the $s$-th moment of the solution,

$$
M_{s}(t)=\int_{\mathbb{R}_{+}} v^{s} f(t ; v) d v
$$

remains bounded for all times. On the other hand, if $\mathbf{S}(s)$ is positive for some $s>1$, then $M_{s}(t)$ diverges exponentially fast as $t \rightarrow \infty$. We exploit this information to prove decay properties to the steady state.

To start with, we note that conservation of the total wealth allows to conclude that at least all moments of order $s \leq 1$ remain uniformly bounded. In fact, by Hölder's inequality,

$$
\int_{\mathbb{R}_{+}} v^{s} f(v) d v \leq\left(\int_{\mathbb{R}_{+}} v f(v) d v\right)^{s} \cdot\left(\int_{\mathbb{R}_{+}} f(v) d v\right)^{1-s}=M_{1}^{s}, \quad 0<s<1 .
$$


Now, let $s>1$ and suppose that the initial density $f_{0}(v)$ satisfies

$$
M_{s}(0)=\int_{\mathbb{R}_{+}} v^{s} f_{0}(v) d v<\infty .
$$

Then, putting $\phi(v)=v^{s}$ in the weak form (2.19), we obtain

$$
\frac{d}{d t} \int_{\mathbb{R}_{+}} v^{s} f(t ; v) d v=\frac{1}{2}\left\langle\int_{\mathbb{R}_{+}^{2}}\left(\sum_{i=1}^{2}\left(p_{i} v+q_{i} w\right)^{s}-v^{s}-w^{s}\right) f(v) f(w) d v d w\right\rangle_{+} .
$$

In the following, we establish upper and lower bounds for the right-hand side of (3.45). These bounds rely on the following elementary inequality.

Lemma 3.3. For arbitrary non-negative real numbers $a$ and $b$, and $s>1$,

$$
\begin{aligned}
& a^{s}+b^{s}+\theta_{s}\left(a^{s-1} b+a b^{s-1}\right) \leq(a+b)^{s} \leq a^{s}+b^{s}+\Theta_{s}\left(a^{s-1} b+a b^{s-1}\right), \\
& \text { with } \theta_{s}=\left\{\begin{array}{ll}
s & (s>3) \\
2^{s-3} s & (2 \leq s \leq 3) \\
0 & (1<s<2)
\end{array} \text { and } \quad \Theta_{s}= \begin{cases}s & (2 \leq s \leq 3) \\
2^{s-3} s & \text { (otherwise })\end{cases} \right.
\end{aligned}
$$

Remark. An investigation of the limit behavior as $a \searrow 0$ and $b>0$ makes clear that $\theta_{s}=0$ for $1<s<2$ cannot be improved in general.

Proof. By homogeneity, it suffices to prove the inequality for $a+b=1$. Define for $s>1$,

$$
\phi(s):=a^{s}+b^{s}+s a b .
$$

A calculation yields $\phi(2)=\phi(3)=1$, independently of $a$ and $b=1-a$. Furthermore, $\phi$ is convex in $s$ since

$$
\phi^{\prime \prime}(s)=a^{s} \ln ^{2} a+b^{s} \ln ^{2} b \geq 0 .
$$

Hence $\phi(s) \leq 1$ if and only if $2 \leq s \leq 3$. Observe that $a^{s-2}+b^{s-2}$ is concave w.r.t. $a=1-b \in(0,1)$ for $2 \leq s \leq 3$, and convex for all other $s>1$; the expression attains its extremal value $2^{3-s}$ at $a=b=\frac{1}{2}$. Hence

$$
a^{s-1} b+a b^{s-1}=a b\left(a^{s-2}+b^{s-2}\right) \begin{cases}\leq 2^{3-s} a b & (2 \leq s \leq 3) \\ \geq 2^{3-s} a b & \text { (otherwise) }\end{cases}
$$

Thus we obtain, for $2 \leq s \leq 3$

$$
a^{s}+b^{s}+2^{s-3} s\left(a^{s-1} b+a b^{s-1}\right) \leq \phi(s) \leq 1=(a+b)^{s},
$$

and with reversed inequalities for $1<s<2$ or $s>3$.

Now let $s>1$ be fixed and consider for $a \in[0,1]$

$$
f_{s}(a):=a^{s}+b^{s}+s\left(a^{s-1} b+a b^{s-1}\right),
$$


with $b=1-a$. Observe that $f_{s}(0)=f_{s}(1)=1$, and furthermore

$$
f_{s}^{\prime}(a)=s(s-1)\left(a^{s-2} b-a b^{s-2}\right),
$$

so that $f_{s}$ has $\bar{a}=\frac{1}{2}$ as the only critical point in $(0,1)$. For $s<3$, one has $f_{s}(\bar{a})=(s+1) 2^{1-s}>1$, so $\bar{a}$ is a maximum point and hence $f_{s}(a) \geq 1$ for $a \in[0,1]$; for $s>3$, it is a minimum point and $f_{s}(a) \leq 1$. Consequently, for $s \leq 3$,

$$
a^{s}+b^{s}+s\left(a^{s-1} b+a b^{s-1}\right)=f_{s}(a) \geq 1=(a+b)^{s} .
$$

The reversed inequality holds for $s \geq 3$.

Using the upper bound in (3.46), estimate

$$
\left(p_{i} v+q_{i} w\right)^{s} \leq p_{i}^{s} v^{s}+q_{i}^{s} w^{s}+\Theta_{s}\left(p_{i}^{s-1} q_{i} v^{s-1} w+p_{i} q_{i}^{s-1} v w^{s-1}\right)
$$

under the integral in (3.45), leading to

$$
\begin{aligned}
\frac{d}{d t} M_{s}(t) \leq & \frac{1}{2}\left\langle\int_{\mathbb{R}_{+}^{2}}\left(\left(p_{1}^{s}+p_{2}^{s}-1\right) v^{s}+\left(q_{1}^{s}+q_{2}^{s}-1\right) w^{s}\right) f(v) f(w) d v d w\right\rangle \\
& +\frac{\Theta_{s}}{2}\left\langle\int _ { \mathbb { R } _ { + } ^ { 2 } } \left(\left(p_{1}^{s-1} q_{1}+p_{2}^{s-1} q_{2}\right) v^{s-1} w\right.\right. \\
& \left.\left.\quad+\left(p_{1} q_{1}^{s-1}+p_{2} q_{2}^{s-1}\right) v w^{s-1}\right) f(v) f(w) d v d w\right\rangle \\
= & \mathbf{S}(s) M_{s}(t)+\Theta_{s}\left\langle p_{i}^{s-1} q_{i}+p_{i} q_{i}^{s-1}\right\rangle_{+} \cdot M_{1}(t) \cdot \int_{\mathbb{R}_{+}} w^{s-1} f(w) d w .
\end{aligned}
$$

Recall that the total wealth $\int v f(v) d v=M_{1}$ is conserved in time. Further, by Hölder's inequality, it follows that

$$
\int_{\mathbb{R}_{+}} w^{s-1} f(w) d w \leq\left(\int_{\mathbb{R}_{+}} w^{s} f(w) d w\right)^{1-\frac{1}{s}} .
$$

Hence, we obtain

$$
\frac{d}{d t} M_{s}(t) \leq \mathbf{S}(s) M_{s}(t)+\Theta_{s} \mathfrak{K}(s) M_{1} \cdot\left(\int_{\mathbb{R}_{+}} v^{s} f(v) d v\right)^{1-\frac{1}{s}},
$$

where

$$
\mathfrak{K}(s):=\left\langle p_{i}^{s-1} q_{i}+p_{i} q_{i}^{s-1}\right\rangle_{+} \leq\left\langle p_{i}^{s}+q_{i}^{s}\right\rangle_{+}=\mathbf{S}(s)+1 .
$$

In particular, if $\mathbf{S}(s)$ is a finite number, then so is $\mathfrak{K}(s)$. In this case, the $s$-th moment grows at most exponentially, with rate $\mathbf{S}(s)$, if it was finite initially. Moreover, if $\mathbf{S}(s)<0$, then the $s$-th moment remains uniformly bounded for 
all times. In fact, an upper bound on the $s$-th moment is determined by the solution of the associated ordinary differential equation

$$
\dot{y}=\mathbf{S} \cdot y+\Theta_{s} \mathfrak{K}(s) M_{1} y^{1-\frac{1}{s}}
$$

with initial condition $y(0)=M_{s}(0)$. The solution is explicitly given by

$$
y(t)=\left[\left(M_{s}(0) \exp \{t \mathbf{S}(s)\}\right)^{1 / s}+\frac{\Theta_{s} \mathfrak{K}(s) M_{1}}{\mathbf{S}(s)}\left(\exp \left\{\frac{t}{s} \mathbf{S}(s)\right\}-1\right)\right]^{s} .
$$

Notice that the first term in the square bracket vanishes for $t \rightarrow \infty$ if $\mathbf{S}(s)<0$, so that the limiting value depends on the initial condition $f_{0}$ only through the total wealth $M_{1}$.

By the same reasoning as above, we construct a bound from below on the time-derivative of the integral. For this, we use the lower bound given in the elementary inequality (3.46). Replacing the respective expressions under the integral, we obtain

$$
\begin{aligned}
\frac{d}{d t} M_{s}(t) \geq & \frac{1}{2} \int_{\mathbb{R}_{+}^{2}}\left(\left\langle p_{1}^{s}+p_{2}^{s}-1\right\rangle v^{s}+\left\langle q_{1}^{s}+q_{2}^{s}-1\right\rangle w^{s}\right) f(v) f(w) d v d w \\
& +\frac{\theta_{s}}{2}\left\langle\int _ { \mathbb { R } _ { + } ^ { 2 } } \left(\left(p_{1}^{s-1} q_{1}+p_{2}^{s-1} q_{2}\right) v^{s-1} w\right.\right. \\
& \left.\left.+\left(p_{1} q_{1}^{s-1}+p_{2} q_{2}^{s-1}\right) v w^{s-1}\right) f(v) f(w) d v d w\right\rangle \\
= & \mathbf{S}(s) M_{s}(t)+\theta_{s} \mathfrak{K}(s) M_{1} \cdot \int_{\mathbb{R}_{+}} w^{s-1} f(w) d w .
\end{aligned}
$$

We use Hölder's inequality to estimate

$$
\int_{\mathbb{R}_{+}} w^{s-1} f(w) d w \geq\left(\int_{\mathbb{R}_{+}} w f(w) d w\right)^{s-1}=M_{1}^{s-1} .
$$

By Gronwall's inequality, a lower bound is given by

$$
M_{s}(t) \geq M_{s}(0) \cdot \exp \{t \cdot \mathbf{S}(s)\}+\frac{\theta_{s} \cdot \mathfrak{K}(s) \cdot M_{1}^{s}}{\mathbf{S}(s)}(\exp \{t \cdot \mathbf{S}(s)\}-1) .
$$

We conclude that if $\mathbf{S}(s)>0$, then the moment $M_{s}$ diverges exponentially in time.

In the special case that $\mathbf{S}(s)=0$, similar but simpler arguments give that the corresponding moment remains either bounded (iff $\mathcal{K}(s)=0$ ) or diverges, but only at the algebraic rate $t^{s}$. Finally, if $\mathbf{S}(s)=+\infty$, an easy argument shows that the $s$-th moment of $f(t ; v)$ must be infinite for all positive times.

Theorem 3.4. [46] Consider a solution $f$ to the Boltzmann equation (2.18). Let $s>1$ be such that $M_{s}(0)=\int_{\mathbb{R}_{+}} v^{s} f_{0}(v) d v<\infty$. 
1. If $0<\mathbf{S}(s)<+\infty$, then the $s$-th moment diverges exponentially fast as $t \rightarrow \infty:$

$$
\begin{aligned}
M_{s}(0)+ & \frac{\theta_{s} \mathfrak{K}(s)}{\mathbf{S}(s)} \cdot M_{1}^{s}+o(1) \\
& \leq \frac{M_{s}(t)}{\exp \{t \cdot \mathbf{S}(s)\}} \leq\left[M_{s}^{1 / s}(0)+\frac{\Theta_{s} \mathfrak{K}(s)}{\mathbf{S}(s)} \cdot M_{1}\right]^{s}+o(1) .
\end{aligned}
$$

2. If $\mathbf{S}(s)<0$, then the $s$-th moment remains uniformly bounded as $t \rightarrow \infty$ :

$$
\frac{\theta_{s} \mathfrak{K}(s)}{|\mathbf{S}(s)|} \cdot M_{1}^{s}+o(1) \leq M_{s}(t) \leq\left(\frac{\Theta_{s} \mathfrak{K}(s)}{|\mathbf{S}(s)|}\right)^{s} \cdot M_{1}^{s}+o(1) .
$$

3. If $\mathbf{S}(s)=0$, then the $s$-th moment either remains bounded or diverges at an algebraic rate:

$$
\left(\frac{\theta_{s} \mathfrak{K}(s)}{s}\right)^{s} \cdot M_{1}^{s}+o(1) \leq t^{-s} \cdot M_{s}(t) \leq\left(\frac{\Theta_{s} \mathfrak{K}(s)}{s}\right)^{s} \cdot M_{1}^{s}+o(1) .
$$

4. Finally, if $\mathbf{S}(s)=+\infty$, then the $s$-th moment is infinite for all $t>0$.

\subsubsection{Existence and tails of the steady state}

The analysis of the previous sections shows that the long-time behavior of solutions is essentially determined by the quantity $\mathbf{S}$. For this reason, let us investigate this function in further detail.

First recall that for an arbitrary non-negative number $p$, the exponential $s \mapsto p^{s}$ is convex in $s>0$. Hence $\mathbf{S}(s)$, which is the average of convex functions, is convex on its domain. By the dominated convergence theorem, $\mathbf{S}(s)$ is welldefined at least for $0<s \leq 1$, but possibly $\mathbf{S}(s)=+\infty$ for all $s>s_{\infty}>1$. Since $\mathbf{S}(1)=0$, convexity leaves only three possibilities for the behavior of $\mathbf{S}$ :

1. $\mathbf{S}(s)$ is non-negative for all $s>0$.

2. $\mathbf{S}(s)$ is negative for some $s \in(0,1)$, and positive for all $s>1$.

3. $\mathbf{S}(s)$ is negative for all $1<s<\bar{s}$, and positive for all $s<1$ and all $s>\bar{s}$; here either $\bar{s}=+\infty$, or $\mathbf{S}(\bar{s})=0$, or $\mathbf{S}(s)=+\infty$ for $s>\bar{s}$.

If $\mathbf{S}$ is differentiable at $s=1$, then the first case corresponds to $\mathbf{S}^{\prime}(1)=0$, the second to $\mathbf{S}^{\prime}(1)>0$, and the last to $\mathbf{S}^{\prime}(1)<0$. These four cases are now discussed in detail.

In the first case, no information about the existence of a long-time limit can be extracted.

In the second case, $r:=-\mathbf{S}(s)>0$ for some $s \in(0,1)$. Observe that $f_{*}=\delta_{0}$, corresponding to a mass concentrated in $v=0$, trivially constitutes a stationary solution of the Boltzmann equation. Recall that the initial condition 
$f_{0}$ is a probability density of finite first moment. Since $s<1$, it follows $K:=$ $d_{s}\left(f_{0}, f_{*}\right)<\infty$. By the contraction estimate, $d_{s}\left(f(t), f_{*}\right) \leq K \exp (-r t) \rightarrow 0$ as $t \rightarrow \infty$. Thus, the solution $f(t)$ converges weakly to $f_{*}$. It is worthwhile to observe that, by Theorem 3.1, all moments $M_{s}(t)$ with $s>1$ diverge as $t \rightarrow \infty$. So, although each $f(t)$ for $t>0$ has the same (positive and finite) first moment as $f_{0}$, one cannot invoke Prokhorov's theorem to conclude that also the weak limit $f_{*}$ has positive first moment.

The third case is the most interesting one. Choose some $s \in(1,2)$ with $s<\bar{s}$; then $r:=-\mathbf{S}(s)>0$. Assume that the initial datum of $f$ possesses a moment of order $S>s$. In view of the completeness of $\left(\mathcal{M}_{M, s}, D_{s}\right)$ when $s \in(1,2)[3]$, the existence of the long-time limit can be concluded directly from the contractivity of the kinetic equation in $D_{s}$-norm (cf. Theorem 3.1).

The same result can be achieved by means of the metric $d_{s}$. In fact, $f(t ; v)$ has the Cauchy property in $d_{s}$; notice that $d_{s}\left[f(t), f_{0}\right]$ is always finite since $s<2$ and the first moment (mean wealth) is conserved under evolution. Moreover, as we required $s<\bar{s}$, there exists a $s^{\prime}$ with $s<s^{\prime}<\min (\bar{s}, S)$; by Theorem 3.4 , the moment of order $s^{\prime}$ remains uniformly bounded. It follows that $f(t)$ converges in $d_{s}$ to a limit distribution $f_{\infty}(v)$, which is normalized and has the same first moment as $f(t)$.

This convergence implies that $f_{\infty}$ is a steady state for the kinetic equation (2.18). Indeed, denote by $f_{\infty}(t)$ the solution to (2.18) with initial datum $f_{\infty}$, then Theorem 3.1 gives

$$
\begin{aligned}
d_{s}\left[f_{\infty}(t), f_{\infty}\right] & \leq d_{s}\left[f_{\infty}(t), f(t+T)\right]+d_{s}\left[f(t+T), f_{\infty}\right] \\
& \leq e^{-r t} d_{s}\left[f_{\infty}, f(T)\right]+d_{s}\left[f(t+T), f_{\infty}\right] .
\end{aligned}
$$

The last expression can be made arbitrarily small by choosing $T$ large enough, so that $f_{\infty}(t)=f_{\infty}$ for all $t \geq 0$. In fact, $f_{\infty}$ is the only steady state with the respective value of the first moment; for if $f_{\infty}^{\prime}$ is another steady state with the same first moment, then $d_{s}\left[f_{\infty}, f_{\infty}^{\prime}\right]$ is finite, and so, invoking Theorem 3.1 again,

$$
d_{s}\left[f_{\infty}, f_{\infty}^{\prime}\right] \leq e^{-r} d_{s}\left[f_{\infty}, f_{\infty}^{\prime}\right]
$$

which forces $f_{\infty}=f_{\infty}^{\prime}$. Finally, consider a solution $f$ which has arbitrary moments bounded initially. Theorem 3.4 gives a time-uniform bound for moments of order less than $\bar{s}$. Since convergence $f(t) \rightarrow f_{\infty}$ in $d_{s}$ implies weak* convergence of the associated measures, it follows that $f_{\infty}$ has finite moments of all orders less than $\bar{s}$. On the other hand, no moment of order larger than $\bar{s}$ can be finite. To see this, simply apply Theorem 3.4 to the steady state solution $f_{\infty}(t) \equiv f_{\infty}$ to derive a contradiction.

We summarize the results of this section.

Theorem 3.5. [46] Let $f(t ; v)$ be the (unique) weak solution of the Boltzmann equation (2.18), which has initially finite moments up to order $S>0$. Further assume that $\mathbf{S}(s)<0$ for some $s \in(0, S)$. Then $f$ converges exponentially fast 
in $D_{s}$ (respectively in $d_{s}$ ) to a steady state $f_{\infty}$,

$$
D_{s}\left[f(t), f_{\infty}\right] \leq D_{s}\left[f_{0}, f_{\infty}\right] \exp \{-|\mathbf{S}(s)| t\} .
$$

If $s<1$, then $f_{\infty}$ is a Dirac distribution centered at $v=0$, and there are no other steady states. If $s>1$, then $f_{\infty}$ has mean wealth equal to $M$, and it is the only steady state with this mean wealth. Moreover, if $\mathbf{S}\left(s^{\prime}\right)<0$ exactly for $1<s^{\prime}<\bar{s}$, possibly with $\bar{s}=+\infty$, then $f_{\infty}$ has finite moments of all orders less than $\bar{s}$, while moments of order larger than $\bar{s}$ are infinite.

\subsubsection{Regularity of the steady wealth distribution}

Even if the most important property of the steady state distribution is its behavior for large values of the wealth variable, which clarifies the eventual formation of Pareto tails, other characteristics can be extracted by a direct investigation of the collision integral. In particular, it is surprising that in most cases one obtains that the steady state is smooth.

Theorem 3.6. [46] Assume that there are positive numbers $r$ and $\delta$ such that

$$
p_{i}^{r}+q_{i}^{r} \geq 1+\delta^{r} \quad \text { a.s. }
$$

Assume further that $\mathbf{S}(s)<0$ for some $s>1$, so that a non-trivial steady state $f_{\infty}$ to the kinetic equation (2.18) exists. Then either $f_{\infty}$ is a Dirac distribution centered at $v=M$, or $f_{\infty}$ is a smooth function and belongs to the $r$-th Gevrey class, i.e.

$$
\left|\hat{f}_{\infty}(\xi)\right| \leq \exp \left(-\mu|\xi|^{r}\right) \quad \text { for }|\xi| \geq \rho,
$$

with suitable positive numbers $\rho$ and $\mu$.

Remark 3.7. Since the mean wealth is preserved, i.e. $\left\langle p_{i}+q_{i}\right\rangle_{+}=1$, it follows that $r<1$ in (3.54). Notice that condition (3.54) imposes no serious restrictions on the distribution of the interaction coefficients. For instance, it allows any probability measure which is compactly supported in the interior of the first quadrant of the $p_{i}-q_{i}$-plane.

The proof of Theorem 3.6 is done on the level of Fourier transforms, and is divided into a series of Lemmas. The first is concerned with the behavior of $\hat{f}_{\infty}$ near the origin:

Lemma 3.8. Under the hypotheses of Theorem 3.6, either $f_{\infty}$ is a Dirac distribution, i.e. $|\hat{f}| \equiv 1$, or there exist positive numbers $\rho$ and $\kappa$ s.t.

$$
\left|\hat{f}_{\infty}(\xi)\right| \leq \exp \left(-\kappa \xi^{2}\right) \quad \text { for }|\xi|<\rho .
$$

Proof. The following proof uses no properties of $f_{\infty}$ other than the finiteness of the first moment. First assume that $f_{\infty}$ also has finite second moment. Then $\hat{f}_{\infty}$ is twice differentiable at $\xi=0$, and

$$
\hat{f}_{\infty}(\xi)=1-i M \xi-\frac{1}{2} Q \xi^{2}+o\left(\xi^{2}\right),
$$


where $M$ and $Q$ denote the first and second moment of $f_{\infty}$, respectively. This means that

$$
\left|\hat{f}_{\infty}(\xi)\right|^{2}=1-\left(Q-M^{2}\right) \xi^{2}+o\left(\xi^{2}\right) .
$$

Now either $Q=M^{2}$, which implies that $f_{\infty}$ is concentrated at $\xi=M$, or $\kappa:=\frac{1}{4}\left(Q-M^{2}\right)>0$ makes $(3.55)$ true for some small $\rho>0$.

In the case that the second moment of $\hat{f}_{\infty}$ is infinite, we write

$$
\hat{f}_{\infty}(\xi)=\int_{\mathbb{R}} f_{\infty}(v) e^{-i \xi v} d v=1-\int_{\mathbb{R}}(1-\cos (\xi v)) d f_{\infty}(v)-i \int_{\mathbb{R}} \sin (\xi v) d f_{\infty}(v),
$$

so that, with the obvious meaning of $H_{1}$ and $H_{2}$,

$$
\left|\hat{f}_{\infty}(\xi)\right|^{2}=1-2 H_{1}(\xi)+H_{1}(\xi)^{2}+H_{2}(\xi)^{2} .
$$

The elementary inequalities $1-\cos x \leq|x|$ and $|\sin x| \leq|x|$ for $x \in \mathbb{R}$ imply

$$
H_{1}(\xi) \leq|\xi| \int_{\mathbb{R}} v d f_{\infty}(v)=M|\xi|, \quad\left|H_{2}(\xi)\right| \leq|\xi| \int_{\mathbb{R}} v d f_{\infty}(v)=M|\xi| .
$$

On the other hand, with the elementary inequalities

$$
1-\cos x \geq \frac{1}{3} x^{2} \quad \text { for }|x| \leq 1, \quad 1-\cos x \geq 0 \quad \text { for } x \in \mathbb{R},
$$

one obtains for arbitrary $\xi \in \mathbb{R}$

$$
H_{1} \geq \frac{\xi^{2}}{3} \int_{|\xi v|<1} v^{2} d f_{\infty}(v)=A(\xi) \xi^{2} .
$$

Here $A(\xi)$ is a positive, non-increasing function with $\lim _{\xi \rightarrow 0} A(\xi)=+\infty$, since the second moment of $f_{\infty}$ diverges. Choosing $\rho>0$ so small that $A(\rho) \geq 2 M^{2}$, it follows that

$$
\left|\hat{f}_{\infty}(\xi)\right|^{2} \leq 1-2 M^{2} \xi^{2} \quad \text { for }|\xi|<\rho .
$$

Hence (3.55) holds with $\kappa:=\frac{1}{2} M^{2}$, possibly after diminishing $\rho$ suitably.

From now on, we will make use of a method first introduced by Bobylev and Cercignani in [12]. Gevrey regularity for the elastic Boltzmann equation for Maxwell molecules has been recently posted in [32]. We introduce the fixed point operator

$$
\mathcal{R}[\psi](\xi):=\left\{\begin{array}{cc}
\hat{f}_{\infty}(\xi) & \text { if }|\xi|<\rho, \\
\left\langle\psi\left(p_{i} \xi\right) \psi\left(q_{i} \xi\right)\right\rangle_{+} & \text {if }|\xi| \geq \rho .
\end{array}\right.
$$

on bounded functions $\psi: \mathbb{R} \rightarrow \mathbb{C}$. Notice that $\mathcal{R}$ is closely related to the Fourier transform of the collision kernel. Furthermore, for $\mu \geq 0$, define $K_{\mu}$ as the set of functions $\psi$ with $\psi(\xi)=\hat{f}_{\infty}(\xi)$ for $|\xi|<\rho$, satisfying the estimate

$$
|\psi(\xi)| \leq \exp \left(-\mu|\xi|^{r}\right) \quad \text { for }|\xi| \geq \rho \text {. }
$$

Here $\rho$ is the constants from Lemma 3.8 above. Eventually, we wish to prove that $\hat{f}_{\infty} \in K_{\mu}$ for a suitable $\mu>0$. 
Lemma 3.9. Under the hypotheses of Theorem 3.6, and assuming that $f_{\infty}$ is not a Dirac distribution, there exists some $\mu>0$, depending only on $\rho$ and $\kappa$ from (3.55), such that $\mathcal{R}$ maps $K_{\mu}$ into itself.

Proof. Let $\psi \in K_{\mu}$ and $\xi \in \mathbb{R}$ with $|\xi| \geq \rho$ be arbitrary. Lemma 3.8 assures that the estimate $(3.55)$ holds with $\psi$ in place of $\hat{f}_{\infty}$. We show that

$$
e^{\mu|\xi|^{r}} \cdot|\mathcal{R}[\psi](\xi)| \leq\left\langle e^{\mu|\xi|^{r}} \cdot\left|\psi\left(p_{i} \xi\right)\right| \cdot\left|\psi\left(q_{i} \xi\right)\right|\right\rangle_{+} \leq 1 .
$$

To this end, we prove that the term inside the expectation value is less/equal one a.s. We distinguish four cases:

1. If both $p_{i}|\xi| \geq \rho$ and $q_{i}|\xi| \geq \rho$, then the hypothesis (3.54) yields

$$
e^{\mu|\xi|^{r}} \cdot\left|\psi\left(p_{i} \xi\right)\right| \cdot\left|\psi\left(q_{i} \xi\right)\right| \leq e^{\mu|\xi|^{r}-\mu p_{i}^{r}|\xi|^{r}-\mu q_{i}^{r}|\xi|^{r}} \leq e^{-\mu|\xi|^{r} \delta^{r}} \leq 1 .
$$

2. If both $p_{i}|\xi|<\rho$ and $q_{i}|\xi|<\rho$, then we conclude from (3.54) that $p_{i}^{2}+q_{i}^{2} \geq$ $2^{1-2 / r}$, so

$$
e^{\mu|\xi|^{r}} \cdot\left|\psi\left(p_{i} \xi\right)\right| \cdot\left|\psi\left(q_{i} \xi\right)\right| \leq e^{\mu|\xi|^{r}-\kappa p_{i}^{2}|\xi|^{2}-\kappa q_{i}^{2}|\xi|^{2}} \leq e^{-|\xi|^{r}\left(\mu-\kappa \cdot 2^{1-2 / r} \rho^{2-r}\right)} \leq 1,
$$

provided that $\mu \leq 2^{1-2 / r} \rho^{2-r} \cdot \kappa$.

3. Now assume that $p_{i}|\xi|<\rho$ while $q_{i}|\xi| \geq \rho$. Using (3.54) once again, one finds

$$
e^{\mu|\xi|^{r}} \cdot\left|\psi\left(p_{i} \xi\right)\right| \cdot\left|\psi\left(q_{i} \xi\right)\right| \leq e^{\mu|\xi|^{r}-\kappa p_{i}^{2}|\xi|^{2}-\mu q_{i}^{r}|\xi|^{r}} \leq e^{\mu\left(p_{i}^{r}-\delta^{r}\right)|\xi|^{r}-\kappa p_{i}^{2}|\xi|^{2}}
$$

Obviously, the last expression is less than one if $p_{i}<\delta$. Assume now that $p_{i} \geq \delta$. Since $|\xi| \geq \rho$, one has $p_{i}|\xi| \geq \delta \rho$ and hence the exponent can be estimated by

$$
\mu p_{i}^{r}|\xi|^{r}-\kappa p_{i}^{2}|\xi|^{2} \leq p_{i}^{r}|\xi|^{r}\left(\mu-\kappa \delta^{2-r} \rho^{2-r}\right) .
$$

The last expression is negative provided that $\mu \leq(\delta \rho)^{2-r} \cdot \kappa$.

4. The last case, $p_{i}|\xi| \geq \rho$ and $q_{i}|\xi|<\rho$, is treated in the same way and yields the same condition on $\mu$.

In summary, (3.57) holds if $\mu$ is the minimum of $2^{1-2 / r} \rho^{2-r} \cdot \kappa$ and $(\delta \rho)^{2-r} \cdot \kappa$.

Lemma 3.10. Under the hypotheses of Theorem 3.6, and assuming that $f_{\infty}$ is not a Dirac distribution, $\hat{f}_{\infty} \in K_{\mu}$ with the $\mu$ from Lemma 3.9 above.

Proof. Let $\psi_{0}(\xi)=\hat{f}_{\infty}(\xi)$ for $|\xi|<\rho$, and $\psi_{0}(\xi)=0$ for $|\xi| \geq \rho$. Define inductively the sequence $\psi_{n+1}=\mathcal{R}\left[\psi_{n}\right]$. By Lemma 3.9, each $\psi_{n}$ belongs to $K_{\mu} \subset K_{0}$. Moreover, by the same estimates that lead to Theorem 3.1, one proves that $\mathcal{R}$ acts as a contraction on $K_{0}$ in the following sense. Clearly $\mathcal{R}$ maps $K_{0}$ into itself, and any two functions $\psi, \varphi \in K_{0}$ satisfy

$$
\frac{|\mathcal{R}[\psi](\xi)-\mathcal{R}[\varphi](\xi)|}{|\xi|^{s}} \leq \sup _{\xi^{\prime} \neq 0}\left(\frac{\left|\psi\left(\xi^{\prime}\right)-\varphi\left(\xi^{\prime}\right)\right|}{\left|\xi^{\prime}\right|^{s}}\right) \cdot\left\langle p_{i}^{s}+q_{i}^{s}\right\rangle_{+} .
$$


The supremum on the right-hand side is obviously finite since $\psi\left(\xi^{\prime}\right)=\varphi\left(\xi^{\prime}\right)=$ $\hat{f}_{\infty}\left(\xi^{\prime}\right)$ for $\left|\xi^{\prime}\right|<\rho$. By hypothesis, $\mathbf{S}(s)<0$, so $0<\left\langle p_{i}^{s}+q_{i}^{s}\right\rangle_{+}<1$, so (3.58) is a genuine contraction estimate and thus implies local uniform convergence of the sequence $\psi_{n}$ to a limit function $\psi_{\infty}$. Local uniform convergence in combination with the pointwise estimates (3.56) is more than enough to invoke Lebesgue's dominated convergence theorem and conclude

$$
\psi_{\infty}(\xi)=\left\langle\psi_{\infty}\left(p_{i} \xi\right) \psi_{\infty}\left(q_{i} \xi\right)\right\rangle_{+}, \quad \text { for }|\xi| \geq \rho,
$$

i.e. $\psi_{\infty}$ is a fixed point of the operator $\mathcal{R}$. It is, in fact, the unique fixed point of $\mathcal{R}$ on $K_{0}$ because of its contractivity (3.58). But clearly $\hat{f}_{\infty} \in K_{0}$ is one fixed point. So $\hat{f}_{\infty}=\psi_{\infty} \in K_{\mu}$.

By definition of $K_{\mu}$, Theorem 3.6 now follows immediately from Lemma 3.10.

\section{Nonconservative models}

A crucial assumption made for the models considered so far is the conservation (at least in a statistical sense) of the average wealth per agent, i.e. the first moment of the wealth distribution, over time. Wealth conservation sounds plausible on a microscopic level, whereas on a macroscopic level, it is arguable that the apparent conservation is in reality a mixture of two effects. On one hand, wealth is created through the production of goods, interests on savings etc. On the other hand, (monetary) wealth is lost through inflation.

Kinetic models which take these two effects into account, were proposed by Slanina [54], and were further developed by Pareschi and one of the authors [50]. In order to incorporate the creation of wealth, the respective trade rules are designed to "reward" agents for trading activity. In the CPT model (3.35), this can be achieved by assuming that the market risk satisfies $\left\langle\eta_{i}\right\rangle=\varepsilon>0$. In other words, the risky investment is more likely to create additional wealth, than to destroy existing wealth. This is a genuine motivation for agents to engage in trades! The effect of inflation is modelled by a time-dependent rescaling $f(t ; w) \leadsto g(t ; v)$ of the wealth distribution,

$$
g(t ; v)=e^{\varepsilon t} f(t ; w), \quad w=e^{\varepsilon t} v,
$$

chosen so that the mean wealth of $g(t ; v)$ is kept constant. The monetary unit is adapted in a way that people stay equally wealthy on the average. The Boltzmann equation (2.18) is respectively modified by an additional drift term,

$$
\frac{\partial}{\partial t} g=Q_{+}(g, g)-g+\varepsilon \frac{\partial}{\partial v}(v g) .
$$

Solutions to this equation have been proven [50] to converge to a steady state $g_{\infty}$, which may or may not have a Pareto tail. Again, the evolution of moments can be analyzed, and leads to a classification of the tail size in terms of properties of the $\eta_{i}$. 


\subsection{Slanina's model}

Motivated by the analogy with a dissipative Maxwell gas, F. Slanina [54] introduced an increasing wealth model where

$$
p_{1}=\lambda, \quad q_{1}=1-\lambda+\epsilon ; \quad p_{2}=1-\lambda+\epsilon, \quad q_{2}=\lambda .
$$

In (4.62), the growth parameter $\epsilon$ is a fixed positive constant, which implies that the total wealth has increased after the trade,

$$
v^{*}+w^{*}=(1+\epsilon)(v+w) .
$$

The aforementioned model is a particular case of the general mixing model introduced in [5], where the collision rules read

$$
v^{*}=p v+q w, \quad w^{*}=q v+p w ; \quad p>q>0 .
$$

Similarly to the $p_{i}$ and $q_{i}$ in (2.17), the interaction parameters $p$ and $q$ determine the agents' behavior in binary trades. It suffices to consider constant $p$ and $q$ instead of random coefficients. Choosing $\phi(v)=v$ in (2.19) shows that

$$
M(t)=\int_{\mathbb{R}^{+}} v f(t ; v) d v=M(0) \exp \{(p+q-1) t\} .
$$

Therefore, unless $p+q=1$, which implies that the model is pointwise conservative, the mean wealth is increasing, if $p+q>1$, or decreasing, if $p+q<1$, exponentially in time. In both cases, however, stationary solutions do not exist. However, the large-time behavior of the model can be described by self-similar solutions.

\subsubsection{Self-similar solutions}

The standard way to look for self-similarity is to scale the solution according to the rule indicated in (4.60). More precisely, define the rescaled solution $g$ by

$$
g(t ; v)=M(t) f(t ; M(t) v),
$$

which implies that $\int v g(t ; v) d v=1$ for all $t \geq 0$. Moreover $g=g(t ; v)$ satisfies the equation

$$
\begin{aligned}
\frac{d}{d t} \int_{\mathbb{R}_{+}} \phi(v) g(t ; v) d v-(p+q-1) & \int_{\mathbb{R}_{+}} \phi(v) \frac{\partial}{\partial v}(v g) d v \\
& =\int_{\mathbb{R}_{+}^{2}} g(v) g(w)\left(\phi\left(v^{*}\right)-\phi(v)\right) d v d w .
\end{aligned}
$$

Choosing $\phi(v)=e^{-s v}$, with $s \in \mathbb{R}_{+}$, gives the representation of equation (4.67) in terms of the Laplace transform $\tilde{g}$ of $g$,

$$
\frac{\partial \tilde{g}}{\partial t}+s(p+q-1) \frac{\partial \tilde{g}}{\partial s}=\tilde{g}(p s) \tilde{g}(q s)-\tilde{g}(s) .
$$


Steady solutions to equation (4.68) satisfy

$$
s(p+q-1) \frac{\partial \tilde{g}}{\partial s}=\tilde{g}(p s) \tilde{g}(q s)-\tilde{g}(s) .
$$

Suppose $\sqrt{p}+\sqrt{q}=1$. Then, since

$$
p+q-1=-\sqrt{2 p} \sqrt{2 q},
$$

direct computations show that the function

$$
\tilde{g}_{\infty}(s)=(1+\sqrt{2 s}) e^{-\sqrt{2 s}}
$$

solves (4.69).

\subsubsection{A Fokker-Planck approximation}

The steady solution (4.70) is independent of the values of $p$ and $q$, provided that $\sqrt{p}+\sqrt{q}=1$. This gives us the possibility to obtain its analytic expression without resorting to the inverse Laplace transform. Let us use a second order Taylor expansion of $\phi\left(v^{*}\right)$ around $v$

$$
\phi\left(v^{*}\right)-\phi(v)=((p-1) v+q w) \phi^{\prime}(v)+\frac{1}{2}((p-1) v+q w)^{2} \phi^{\prime \prime}(\tilde{v}),
$$

where, for some $0 \leq \theta \leq 1$,

$$
\tilde{v}=\theta v^{*}+(1-\theta) v .
$$

Inserting this expansion in the collision operator, we obtain the equality

$$
\begin{aligned}
& \frac{d}{d t} \int_{\mathbb{R}_{+}} \phi(v) g(t ; v) d v+q \int_{\mathbb{R}_{+}} \phi^{\prime}(v)(v-1) g(v) d v \\
= & \frac{1}{2} \int_{\mathbb{R}_{+}} g(v)\left((p-1)^{2} v^{2}+q^{2} w^{2}+2(p-1) q v w\right) \phi^{\prime \prime}(v) d v+R(p, q),
\end{aligned}
$$

where

$$
R(p, q)=\frac{1}{2} \int_{\mathbb{R}_{+}^{2}}((p-1) v+q w)^{2}\left(\phi^{\prime \prime}(\tilde{v})-\phi^{\prime \prime}(v)\right) g(v) g(w) d v d w .
$$

For small values of the parameter $q$, let us set

$$
\tau=q t, \quad h(\tau ; v)=g(t ; v),
$$

which implies that $h(v, \tau)$ satisfies the equation

$$
\begin{aligned}
& \frac{d}{d \tau} \int_{\mathbb{R}_{+}} \phi(v) h(\tau ; v) d v+\int_{\mathbb{R}_{+}} \phi^{\prime}(v)(v-1) h(v) d v \\
= & \frac{1}{2} \int_{\mathbb{R}_{+}} h(v) \frac{(p-1)^{2}}{q} v^{2} \phi^{\prime \prime}(v) d v+R_{1}(p, q),
\end{aligned}
$$


where the remainder $R_{1}$ is given by

$$
R_{1}(p, q)=\frac{1}{2} \int_{\mathbb{R}_{+}}\left(q w^{2}+2(p-1) v w\right) \phi^{\prime \prime}(v) d v+\frac{1}{q} R(p, q) .
$$

Since equation (4.73) coincides with the original Boltzmann equation, if $p$ and $q$ satisfy the condition $\sqrt{p}+\sqrt{q}=1$, the function (4.70) remains a stationary solution. On the other hand, under the same conditions on the parameters, $p=(1-\sqrt{q})^{2}$ implies that

$$
\lim _{q \rightarrow 0} \frac{(p-1)^{2}}{q}=4
$$

Then, equation (4.73) is well-approximated by

$$
\frac{d}{d \tau} \int_{\mathbb{R}_{+}} \phi(v) h(\tau ; v) d v+\int_{\mathbb{R}_{+}} \phi^{\prime}(v)(v-1) h(v) d v=2 \int_{\mathbb{R}_{+}} h(v) v^{2} \phi^{\prime \prime}(v) d v .
$$

Equation (4.75) is nothing but the weak form of the Fokker-Planck equation

$$
\frac{\partial h}{\partial \tau}=2 \frac{\partial^{2}}{\partial v^{2}}\left(v^{2} h\right)+\frac{\partial}{\partial v}((v-1) h),
$$

which admits a unique stationary state of unit mass, given by the $\Gamma$-distribution $[14,29]$

$$
P_{\infty}(v)=\frac{1}{\sqrt{2 \pi}} \frac{\exp \left(-\frac{1}{2 v}\right)}{v^{5 / 2}} .
$$

This stationary distribution exhibits a Pareto power law tail for large $v$ 's. From the asymptotic equivalence of the Boltzmann equation (4.67) with the FokkerPlanck equation (4.75), and from the invariance of the steady state, we can conclude that $P_{\infty}(v)$ has Laplace transform $\tilde{g}(s)$, and for this reason is a steady state of the Boltzmann equation in case $\sqrt{p}+\sqrt{q}=1$. In this case, however, the quantity $p+q-1$, which can produce exponential growth of wealth (when positive), or exponential dissipation of wealth (when negative), is negative. This is quite remarkable since it shows that this uneven distribution of wealth which characterizes most western economies may not only be produced as the effect of a growing economy but also under critical economical circumstances. This simple example, in addition, shows that the old intuition of Amoroso [1] about the shape of wealth distribution was exact.

\subsubsection{Fokker-Planck equations}

Apart from an investigation of moments, the Boltzmann equations (2.18) or (4.61) are hard to analyze, even in the stationary regime. The method of the previous section provides a useful way to generate from the kinetic equation a Fokker-Planck equation, i.e. a parabolic differential equation of second order, which is better accessible. 
This asymptotic procedure, which has been used in Section 4.1.2 in the case of nonconservative economies, still applies to conservative (in the mean) models. Consider the CPT model (3.35) with saving propensity $\lambda=1-\beta^{2}$ and market risks $\beta \eta_{i}$,

$$
v^{*}=\left(1-\beta^{2}\right) v+\beta \eta_{1} v+\beta^{2} w, \quad w^{*}=\left(1-\beta^{2}\right) w+\beta \eta_{2} w+\beta^{2} v .
$$

where $\beta>0$ is a small parameter, and $\eta_{1}$ and $\eta_{2}$ are two equally distributed, centered random variables with $\sigma^{2}:=\left\langle\eta_{i}^{2}\right\rangle$. Expanding the collisional operator in terms of $\beta$,

$$
\begin{aligned}
& Q_{+}(f, f)[\varphi]-\int_{\mathbb{R}_{+}} \varphi(v) d v \\
=\int_{\mathbb{R}_{+}^{2}}\left(\varphi^{\prime}(v)\left[\beta^{2}(w-v)+\beta\langle\eta\rangle v\right]\right. & \\
& \left.\quad+\frac{1}{2} \varphi^{\prime \prime}(v)\left[\beta^{2}(w-v)+\beta\langle\eta\rangle\right]^{2}+O\left(\beta^{4}\right)\right) f(v) f(w) d v d w \\
= & \beta^{2} \int_{\mathbb{R}_{+}} \varphi(v)\left(-\frac{\partial}{\partial v}[(M-v) f(v)]+\frac{\sigma^{2}}{2} \frac{\partial^{2}}{\partial v^{2}}\left[v^{2} f(v)\right]\right) d v+O\left(\beta^{4}\right) .
\end{aligned}
$$

Finally, increase the collision frequency by rescaling $t \leadsto t / \beta^{2}$. In the limit $\beta \rightarrow 0$, the Boltzmann equation turns into the Fokker-Planck equation

$$
\frac{\partial}{\partial t} f=\frac{\sigma^{2}}{2} \frac{\partial^{2}}{\partial v^{2}}\left[v^{2} f\right]+\frac{\partial}{\partial v}[(v-M) f]
$$

which possesses an explicit stationary solution of Amoroso type [1]

$$
f_{\infty}(v)=C_{\sigma, M} \exp \left(-\frac{2 M}{\sigma^{2} v}\right) v^{-\left(2+2 / \sigma^{2}\right)} .
$$

The solution $f_{\infty}$ constitutes an approximation of the steady state of the respective (kinetic) CPT model for sufficiently small $\beta>0$ [50]. For instance, in agreement with results on the CPT model, more risky trades (larger $\sigma$ ) induce fatter Pareto tails (decreasing index $\alpha=1+2 / \sigma^{2}$ ).

\subsection{Large-time behavior of nonconservative economies}

The analysis of Sections 3.4, can be easily extended to equation (2.18), even in the case of nonconservative trades. In particular, Theorems 3.1 and 3.2 remain valid, and insure the existence of a unique solution to the Boltzmann equation for the density $f$. The real problem, on the other hand, is related to the scaled density $g(t ; w)$, which satisfies equation (4.67).

\subsubsection{Convergence to self-similar solutions}

In consequence of the scaling property (2.16)

$$
\widehat{g}(\xi)=\widehat{g}\left(\frac{\xi}{M(t)}\right)
$$


from (3.42) we obtain that the solution to equation (4.67) satisfies the bound

$$
d_{s}\left[g_{1}(t), g_{2}(t)\right]=\sup _{\xi \in \mathbb{R}} \frac{\left|\widehat{g_{1}}(t ; \xi)-\widehat{g_{2}}(t ; \xi)\right|}{|\xi|^{s}}=\left(\frac{1}{M(t)}\right)^{s} d_{s}\left[f_{1}(t), f_{2}(t)\right] .
$$

It follows that, if $g_{1}(t)$ and $g_{2}(t)$ are two solutions of the scaled Boltzmann equation (4.67), corresponding to initial values $f_{1,0}$ and $f_{2,0}$ in $\mathcal{M}_{M, s}$, for some $1 \leq s \leq 2$, for all times $t \geq 0$,

$$
d_{s}\left[g_{1}(t), g_{2}(t)\right] \leq \exp \left\{\left[\left(p^{s}+q^{s}-1\right)-s(p+q-1)\right] t\right\} d_{s}\left[f_{1,0}, f_{2,0}\right] .
$$

Let us define, for $s \geq 1$,

$$
\mathbf{R}_{p, q}(s)=p^{s}+q^{s}-1-s(p+q-1) .
$$

Then, the sign of $\mathbf{R}_{p, q}$ now determines the asymptotic behavior of the distance $d_{s}\left[g_{1}(t), g_{2}(t)\right]$. We give below the main result which characterize the sign of the function (4.83).

Lemma 4.1. There exists some $\bar{s} \in(0,+\infty]$ such that $\mathbf{R}_{p, q}(s)<0$ for all $1<s<\bar{s}$. If $p+q \geq 1$, but both $p, q \leq 1$, then $\bar{s}=+\infty$, while $\bar{s}$ is finite in all other cases.

The main consequence of Lemma 4.1 is the following theorem.

Theorem 4.2. [50] Let $g_{1}(t)$ and $g_{2}(t)$ be two solutions of the Boltzmann equation (4.67), corresponding to initial values $f_{1,0}$ and $f_{2,0}$ in $\mathcal{M}_{M, s}$, for some $1 \leq s \leq 2$. There exists a constant $\bar{s}>1$ such that, if $1<s<\bar{s}$, then

$$
d_{s}\left[g_{1}(t), g_{2}(t)\right] \leq \exp \left\{-C_{s} t\right\} d_{s}\left[f_{1,0}, f_{2,0}\right]
$$

for all times $t \geq 0$. The constant $C_{s}=-\mathbf{R}_{p, q}(s)$ is strictly positive, so that the distance $d_{s}$ is contracting exponentially in time.

Existence and uniqueness of the stationary solution to equation (4.67) follow along the same lines of Section 3.4, using now as convex function $\mathbf{R}_{p, q}$ instead of $\mathbf{S}$. The main result is now stated in the following theorem.

Theorem 4.3. [50] Let $s>1$ be such that $\mathbf{R}_{p, q}(s)<0$, and let $g_{\infty}(v)$ be the unique stationary solution (of given mean wealth $M>0$ ) to the Boltzmann equation (4.67). Let $g(t ; v)$ be the weak solution of equation (4.67) corresponding to the initial density $f_{0} \in \mathcal{M}_{M, s}$. Then

$$
\int_{\mathbb{R}_{+}} v^{s} g(t ; v) d v \leq c_{\bar{s}}<\infty,
$$

with a time-uniform constant $c_{\bar{s}}$ depending only on $p$ and $q$. Moreover, $g(t ; v)$ converges exponentially fast in Fourier metric towards stationarity,

$$
d_{s}\left[g(t), g_{\infty}\right] \leq d_{s}\left[f_{0}, g_{\infty}\right] \exp \left\{-\left|\mathbf{R}_{p, q}(s)\right| t\right\},
$$

where $\mathbf{R}_{p, q}(s)$ has been defined in (4.83). 
Depending on the values of the mixing parameters $p$ and $q$, the stationary solution $g_{\infty}$ may have overpopulated tails. The Fourier transform of $g_{\infty}$ satisfies

$$
-(p+q-1) \xi \frac{\partial \widehat{g}_{\infty}}{\partial \xi}+\widehat{g}_{\infty}(\xi)=\widehat{g}_{\infty}(p \xi) \widehat{g}_{\infty}(q \xi) .
$$

In the following, let $\bar{s} \in(1,2)$. We would like to decide if the stationary solution $g_{\infty}$ has a fat tail of Pareto index $\bar{s}$. More restrictively, we ask if $g_{\infty}$ belongs to every $\mathcal{M}_{M, s}$ for $s<\bar{s}$, but not to $\mathcal{M}_{M, \bar{s}}$. If this is the case, then its Fourier transform satisfies

$$
\widehat{g}_{\infty}(\xi)=1-i \xi+A|\xi|^{\bar{s}}+o\left(|\xi|^{\bar{s}}\right) .
$$

Substitution of the expansion (4.87) into equation (4.86) shows that the coefficient of the power $|\xi|^{\bar{s}}$ is $A \mathbf{R}_{p, q}(\bar{s})$. Thus, the term $A|\xi|^{\bar{s}}$ can appear in the expansion of $\widehat{g}(\xi)$ as soon as $\mathbf{R}_{p, q}(\bar{s})=0$. As before, tails in the stationary distributions are present in all cases in which there exists a $\bar{s} \in(1,2)$ with $\mathbf{R}_{p, q}(\bar{s})=0$. With slight modifications, the argument also works for $\bar{s} \geq 1$. Thus, the question of existence of a fat tailed steady state is answered by Lemma 4.1.

To finish the discussion, we mention that the passage to the Fokker-Planck equation can be made rigorous.

Theorem 4.4. [50] Let an initial condition $f_{0} \in \mathcal{M}_{M, s}$ be given, with $M>$ 0 and $s>1$. Consider the corresponding family of solutions $h^{(q)}(t)$ to the Boltzmann equation (4.73) for the scaled densities $h^{(q)}(\tau ; v)=g^{(q)}(t ; v)$, with $\tau=q t$. The super-index $(q)$ refers to the mixing parameter $q>0$, and

$$
(p-1)^{2}=\lambda q,
$$

for some fixed $\lambda>0$. Then, as $q \rightarrow 0$, the solutions $h^{(q)}(\tau ; v)$ converge weakly to a limit $h(\tau ; w)$. The latter constitutes a weak solution of the Fokker-Planck equation

$$
\frac{\partial h}{\partial \tau}=\frac{\lambda}{2} \frac{\partial^{2}}{\partial v^{2}}\left(v^{2} h\right)+\frac{\partial}{\partial v}((v-1) h) .
$$

\subsubsection{Regularity of self-similar solutions}

The Fourier transform of the steady solution $g_{\infty}$ of the scaled Boltzmann equation (4.67) solves (4.86). The regularity of $g_{\infty}$ can be recovered from the results of Section 3.4 .4 by rewriting equation (4.86) in an interesting way, which has been proposed by Bobylev and Cercignani [12]. With the definitions

$$
r:=1 /(p+q-1) \quad \text { and } \quad F[\psi](\xi):=\psi(p \xi) \psi(q \xi),
$$

equation (4.86) takes the form

$$
r^{-1} \xi \widehat{g}_{\infty}^{\prime}(\xi)+F\left[\widehat{g}_{\infty}\right](\xi)-\widehat{g}_{\infty}(\xi)=0 .
$$

Equation (4.89) can be rewritten as

$$
\frac{d}{d \xi}\left(\xi^{-r} \widehat{g}_{\infty}(\xi)\right)=-\frac{r}{\xi^{r+1}} F\left[\widehat{g}_{\infty}\right](\xi) .
$$


In analogy to Section 3.4.4, we shall represent $\widehat{g}_{\infty}$ as solution to a fixed-point equation,

$$
\widehat{g}_{\infty}=\mathcal{R}\left[\widehat{g}_{\infty}\right],
$$

where the integral operator $\mathcal{R}$ is now defined by

$$
\mathcal{R}[\psi](\xi):=r \xi^{r} \int_{\xi}^{\infty} \frac{F[\psi](\eta)}{\eta^{r+1}} d \eta=r \int_{1}^{\infty} \frac{F[\psi](\tau \xi)}{\tau^{r+1}} d \tau .
$$

The function $\theta$ with $\theta(\tau)=\frac{r}{\tau^{r+1}}$ for $\tau \geq 1$, and $\theta(\tau)=0$ for $\tau<1$ is a probability density function,

$$
\int_{\mathbb{R}^{+}} \theta(\tau) d \tau=\int_{1}^{\infty} \frac{r}{\tau^{r+1}} d \tau=1 .
$$

Therefore, recalling the definition of $F$, the fixed point equation (4.91) takes the form

$$
\widehat{g}_{\infty}(\xi)=\left\langle\widehat{g}_{\infty}(\tilde{p} \xi) \widehat{g}_{\infty}(\tilde{q} \xi)\right\rangle,
$$

where now $\tilde{p}=\Theta p$ and $\tilde{q}=\Theta q$ are random variables and $\Theta$ is distributed on $\mathbb{R}_{+}$with density $\theta(\tau)$. Thus, the results of Section 3.4.4 apply. In particular, since $\Theta \geq 1$, condition (3.54) is always satisfied for some $r>0$.

Theorem 4.5. Let $r>0$ be such that the mixing parameters satisfy condition (3.54). Denote by $g_{\infty}$ the non-trivial steady state to the kinetic equation (4.61) of given mean $M>0$. Then $g_{\infty}$ is a smooth function and belongs to the $r$-th Gevrey class, i.e.

$$
\left|g_{\infty}(\xi)\right| \leq \exp \left(-\mu|\xi|^{r}\right) \quad \text { for }|\xi| \geq \rho
$$

with suitable positive numbers $\rho$ and $\mu$.

\section{$5 \quad$ Kinetic models for groups of traders}

In this section we propose a generalization of the CPT model, where agents from $n$ different countries or social groups of individuals trade with each other. These groups shall be identified with countries or social classes inside a country. We will adopt the hypothesis that all agents belonging to one group share a common saving rate parameter. This hypothesis can be further relaxed by assuming that the saving rate is a random quantity, with a statistical mean which is different for different social groups. Here we describe the model proposed in [37] which is based on CPT conservative model. A related problem, based on increasing wealth, has been recently introduced and numerically studied in [27]. 


\subsection{The analogy with gas mixtures}

This can be seen as the analogue to the physical problem of a mixture of gases, where the molecules of the different gases exchange momentum in collisions [13]. When two agents from the same country with pre-trade wealths $v$ and $w$ interact - a domestic trade event - , then their post-trade wealths $v^{*}$ and $w^{*}$ are supposed to be given by (3.35) with a common saving rate parameter which is characteristic for this country. On the other hand, in case of an international trade, i.e. when two agents of different countries interact, we assume that each agent uses the transaction parameter which is characteristic for his country. Hence, when two agents, one from country $i(i=1,2, \ldots, n)$ with pre-trade wealth $v$ and the other from country $j(j=1,2, \ldots, n)$ with pre-trade wealth $w$ interact, their post-trade wealths $v^{*}$ and $w^{*}$ are given by

$$
\begin{aligned}
v^{*} & =\left(1-\gamma_{i} \gamma\right) v+\gamma_{j} \gamma w+\eta_{i j} v, \\
w^{*} & =\left(1-\gamma_{j} \gamma\right) w+\gamma_{i} \gamma v+\eta_{j i} w .
\end{aligned}
$$

In (5.93), the trade depends on the transaction parameters $\gamma$ and $\gamma_{i}(i=$ $1, \ldots, n)$, while the risks of the market are described by $\eta_{i j}(i, j=1, \ldots, n)$, which are equally distributed random variables with zero mean and variance $\sigma_{i j}^{2}$. The different variances for domestic trades in each country and for international trades reflect different risk structures in these trades. For example, investments and trades inside different countries or markets may be subject to different types and quantities of risk, and international trading may face additional risks compared to domestic trades.

The trading rule (5.93) preserves, as in the original conservative CPT model, the total wealth in the statistical mean

$$
\left\langle v^{*}+w^{*}\right\rangle=\left(1+\left\langle\eta_{i j}\right\rangle\right) v+\left(1+\left\langle\eta_{j i}\right\rangle\right) w=v+w .
$$

In this setting, we are led to study the evolution of the distribution function for each country as a function depending on the wealth $w \in \mathbb{R}_{+}$and time $t \in \mathbb{R}_{+}, f_{i}=f_{i}(t ; w)$. In analogy with the classical kinetic theory of mixtures of rarefied gases, the time-evolution of the distributions will obey a system of $n$ Boltzmann-like equations, given by

$$
\frac{\partial}{\partial t} f_{i}(t ; w)=\sum_{j=1}^{n} \frac{1}{\tau_{i j}} \mathcal{Q}\left(f_{i}, f_{j}\right)(w), \quad i=1, \ldots, n .
$$

Herein, $\tau_{i j}$ are suitable relaxation times, which depend on the velocity of money circulation [58]. The Boltzmann-like collision operators are derived by standard methods of kinetic theory, considering that the change in time of $f_{i}(t ; w)$ due to binary trades depends on a balance between the gain and loss of agents with wealth $w[29]$. The $\mathcal{Q}$ operator now reads

$$
\mathcal{Q}\left(f_{i}, f_{j}\right)(w)=\left\langle\int_{\mathbb{R}_{+}}\left(\frac{1}{J_{i j}} f_{i}\left(v_{*}\right) f_{j}\left(w_{*}\right)-f_{i}(v) f_{j}(w)\right) d v\right\rangle .
$$


In $(5.96),\left(v_{*}, w_{*}\right)$ denote the pre-trade pair that produces the post-trade pair $(v, w)$, following rules like (5.93), while $J_{i j}$ denotes the Jacobian of the transformation of $(v, w)$ into $\left(v^{*}, w^{*}\right)$. As before, we can fruitfully consider the weak form

$$
\int_{\mathbb{R}_{+}} \mathcal{Q}\left(f_{i}, f_{j}\right)(w) \phi(w) d w=\left\langle\int_{\mathbb{R}_{+}^{2}}\left(\phi\left(v^{*}\right)-\phi(v)\right) f_{i}(v) f_{j}(w) d v d w\right\rangle .
$$

\subsection{A related system of Fokker-Planck equations}

As briefly remarked in Section 4.1.3, it is rather difficult to describe analytically the behavior of the solution to the kinetic system (5.95). By adopting the asymptotic procedure sketched in Section 4.1.3, we can reduce our system to a system of Fokker-Planck type equations. By means of this approach it is easier to identify steady states while retaining important information on the microscopic interaction at a macroscopic level. In the present case, this asymptotic procedure corresponds to consider the joint limits $\gamma \rightarrow 0, \sigma_{i j}^{2} \rightarrow 0$ and $\sigma_{i j}^{2} / \gamma \rightarrow \mu_{i j}$.

The weak form of $(5.95)$ is given by $(i=1, \ldots, n)$

$$
\frac{d}{d t} \int_{\mathbb{R}_{+}} f_{i}(t ; w) \phi(w) d w=\int_{\mathbb{R}_{+}} \sum_{j=1}^{n} \frac{1}{\tau_{i j}} \mathcal{Q}\left(f_{i}, f_{j}\right)(w) \phi(w) d w
$$

where the terms on right hand side are given by (5.97). To study the situation for large times, i.e. close to the steady state, we introduce for $\gamma \ll 1$ the transformation

$$
\tau=\gamma t, \quad g_{i}(\tau ; w)=f_{i}(t ; w), \quad(i=1, \ldots, n) .
$$

This implies $f_{i, 0}=g_{i, 0}$ and the evolution of the scaled densities $g_{i}(\tau ; w)$ follows $(i=1, \ldots, n)$

$$
\frac{d}{d \tau} \int_{\mathbb{R}_{+}} g_{i}(\tau ; w) \phi(w) d w=\frac{1}{\gamma} \int_{\mathbb{R}_{+}} \sum_{j=1}^{n} \frac{1}{\tau_{i j}} \mathcal{Q}\left(f_{i}, f_{j}\right)(w) \phi(w) d w .
$$

By the trading rule (5.93), it holds

$$
v^{*}-v=\gamma\left(\gamma_{j} w-\gamma_{i} v\right)+\eta_{i j} v
$$

Using a second order Taylor expansion of $\phi$ around $v$, we obtain

$$
\begin{aligned}
& \quad \phi\left(v^{*}\right)-\phi(v)=\phi^{\prime}(v)\left[\gamma\left(\gamma_{j} w-\gamma_{i} v\right)+\eta_{i j} v\right]+\frac{1}{2} \phi^{\prime \prime}(\tilde{v})\left[\gamma\left(\gamma_{j} w-\gamma_{i} v\right)+\eta_{i j} v\right]^{2}, \\
& \text { with } \tilde{v}=\theta v^{\prime}+(1-\theta) v \text { for some } 0 \leq \theta \leq 1
\end{aligned}
$$


Inserting this expansion into the collision operators yields

$$
\begin{aligned}
\frac{d}{d \tau} \int_{\mathbb{R}_{+}} g_{i}(\tau ; w) \phi(w) d w & \\
=\frac{1}{\gamma} \sum_{j=1}^{n} \frac{1}{\tau_{i j}}\left\langle\int _ { \mathbb { R } _ { + } ^ { 2 } } \left(\phi ^ { \prime } ( v ) \left[\gamma\left(\gamma_{j} w-\gamma_{i} v\right)\right.\right.\right. & \left.\left.+\eta_{i j} v\right]+\frac{1}{2} \phi^{\prime \prime}(v)\left[\gamma\left(\gamma_{j} w-\gamma_{i} v\right)+\eta_{i j} v\right]^{2}\right) \times \\
& \left.\times g_{i}(\tau ; v) g_{j}(\tau ; w) d v d w\right\rangle+R\left(\gamma, \sigma_{i j}\right),
\end{aligned}
$$

where the remainder $R\left(\gamma, \sigma_{i j}\right)$ converges to zero as $\gamma, \sigma_{i j} \rightarrow 0$, in such a way that $\sigma_{i j}^{2} \rightarrow \mu_{i j} \gamma$, provided further hypotheses on the random variables $\eta_{i j}$ and on $\phi$ hold (cf. [29]).

Recalling that $\left\langle\eta_{i j}\right\rangle=0$ and $\left\langle\eta_{i j}^{2}\right\rangle=\sigma_{i j}^{2}$, in the same limit process we obtain

$$
\begin{gathered}
\lim _{\gamma \rightarrow 0} \frac{1}{\gamma} \sum_{j=1}^{n} \frac{1}{\tau_{i j}}\left\langle\int_{\mathbb{R}_{+}^{2}}\left(\phi^{\prime}(v)\left[\gamma\left(\gamma_{j} w-\gamma_{i} v\right)+\eta_{i j} v\right]+\frac{1}{2} \phi^{\prime \prime}(v)\left[\gamma\left(\gamma_{j} w-\gamma_{i} v\right)+\eta_{i j} v\right]^{2}\right) \times\right. \\
\left.\times g_{i}(\tau ; v) g_{j}(\tau ; w) d v d w\right\rangle \\
=\sum_{j=1}^{n} \frac{1}{\tau_{i j}} \int_{\mathbb{R}_{+}}\left[\phi^{\prime}(v)\left(\gamma_{j} m_{j}-\gamma_{i} v \rho_{j}\right)+\frac{\mu_{i j}}{2} v^{2} \rho_{j} \phi^{\prime \prime}(v)\right] g_{i}(\tau ; v) d v, \quad
\end{gathered}
$$

where

$$
\rho_{j}(\tau)=\int_{\mathbb{R}_{+}} g_{j}(\tau ; w) d w, \quad m_{j}(\tau)=\int_{\mathbb{R}_{+}} w g_{j}(\tau ; w) d w
$$

denote the total population and the total wealth of the $j$-th country, respectively. This expression is nothing but the right hand side of the weak form of the system of Fokker-Planck equations $(i=1, \ldots, n)$

$$
\frac{\partial g_{i}}{\partial \tau}=\sum_{j=1}^{n}\left[\frac{\mu_{i j}}{2 \tau_{i j}} \frac{\partial^{2}}{\partial v^{2}}\left(v^{2} \rho_{j} g_{i}\right)+\frac{1}{\tau_{i j}} \frac{\partial}{\partial v}\left(\left(\gamma_{i} v \rho_{j}-\gamma_{j} m_{j}\right) g_{i}\right)\right] .
$$

To formalize the above, let us introduce some notation. Let $\mathcal{M}_{0}$ be the space of all probability measures in $\mathbb{R}_{+}$and

$$
\mathcal{M}_{p}=\left\{\Psi \in \mathcal{M}_{0}: \int_{\mathbb{R}_{+}} \vartheta^{p} \Psi(\vartheta) d \vartheta<+\infty, p \geq 0\right\},
$$

the space of all Borel probability measures of finite momentum of order $p$, equipped with the topology of the weak convergence of measures.

Let $\mathcal{F}_{p}\left(\mathbb{R}_{+}\right), p>1$ be the class of all real functions $h$ on $\mathbb{R}_{+}$such that $h(0)=h^{\prime}(0)=0$ and $h^{(m)}(v)$ is Hölder continuous of order $\delta$,

$$
\left\|h^{(m)}\right\|_{\delta}=\sup _{v \neq w} \frac{\left|h^{(m)}(v)-h^{(m)}(w)\right|}{|v-w|^{\delta}}<\infty,
$$


the integer $m$ and the number $0<\delta \leq 1$ are such that $m+\delta=p$, and $h^{(m)}$ denotes the $m$-th derivative of $h$.

Using the same ideas of [29], we obtain in this case the following

Theorem 5.1. [37] Let initial probability densities $f_{0, i} \in \mathcal{M}_{M, p}$, where $M>0$ and $p>2$, be given, and assume that the symmetric random variables $\eta_{i j}$ have a density in $\mathcal{M}_{s}$ with $s>p$. Consider the family - parameterized by $\gamma>0-$ of weak solutions $g_{i}^{(\gamma)}$ of the Boltzmann system (5.95) for the scaled densities $g_{i}^{(\gamma)}(\tau ; w)=f_{i}^{(\gamma)}(t ; w)$ with $\tau=\gamma t$.

Then, as $\gamma \rightarrow 0$ and $\sigma_{i j} \rightarrow 0$ in such a way that $\sigma_{i j}=\mu_{i j} \gamma$, the weak solutions converge, up to extraction of a subsequence, to probability densities $g_{i}(\tau ; w)$. These densities constitute weak solutions of the Fokker-Planck system $(5.105)$.

The (relatively) easy structure of the Fokker-Planck system (5.105) allows to study in some simple case the evolution of the mean wealth in each community, as well as the steady state solution. For the sake of simplicity, we will limit ourselves to the case of two populations, in which $\mu_{i j}=\tau_{i j}=1$ for $i=1,2$. From the equations (5.105) it follows that the masses $\rho_{1}(\tau)$ and $\rho_{2}(\tau)$ do not vary with time, while the total wealths $m_{1}(\tau)$ and $m_{2}(\tau)$ satisfy the system of ordinary differential equations

$$
\begin{aligned}
\frac{d m_{1}}{d \tau} & =-\left(\gamma_{1} \rho_{2} m_{1}-\gamma_{2} \rho_{1} m_{2}\right) \\
\frac{d m_{2}}{d \tau} & =+\left(\gamma_{1} \rho_{2} m_{1}-\gamma_{2} \rho_{1} m_{2}\right)
\end{aligned}
$$

System (5.108) can be solved exactly, to give

$$
\begin{aligned}
& m_{1}(\tau)=m_{1}(0) e^{-\left(\gamma_{1} \rho_{2}+\gamma_{2} \rho_{1}\right) \tau}+\frac{\gamma_{2} \rho_{1}}{\gamma_{1} \rho_{2}+\gamma_{2} \rho_{1}}\left(1-e^{-\left(\gamma_{1} \rho_{2}+\gamma_{2} \rho_{1}\right) \tau}\right) \\
& m_{2}(\tau)=m_{2}(0) e^{-\left(\gamma_{1} \rho_{2}+\gamma_{2} \rho_{1}\right) \tau}+\frac{\gamma_{1} \rho_{2}}{\gamma_{1} \rho_{2}+\gamma_{2} \rho_{1}}\left(1-e^{-\left(\gamma_{1} \rho_{2}+\gamma_{2} \rho_{1}\right) \tau}\right)
\end{aligned}
$$

Choosing equally large populations, $\rho_{1}(0)=\rho_{2}(0)$, it follows from formulas (5.109) that the total wealths $m_{1}(\tau)$ and $m_{2}(\tau)$ converge at an exponential rate towards steady values $m_{1, \infty}$ and $m_{2, \infty}$. The latter are inversely proportional to the $\gamma$ 's (a bigger $\gamma$ produces a smaller mean wealth),

$$
m_{1, \infty}=\frac{\gamma_{2}}{\gamma_{1}+\gamma_{2}} ; \quad m_{2, \infty}=\frac{\gamma_{1}}{\gamma_{1}+\gamma_{2}} .
$$

This effect can be directly deduced by examining the analytic expressions of the steady states, given by

$$
\begin{aligned}
& g_{1, \infty}(v)=\frac{c_{1}}{\rho} \frac{1}{v^{2+2 \gamma_{1} / \lambda}} \exp \left\{-\frac{2\left(\gamma_{1} m_{1}+\gamma_{2} m_{2}\right)}{\lambda \rho v}\right\} \\
& g_{2, \infty}(v)=\frac{c_{2}}{\rho} \frac{1}{v^{2+2 \gamma_{2} / \lambda}} \exp \left\{-\frac{2\left(\gamma_{1} m_{1}+\gamma_{2} m_{2}\right)}{\lambda \rho v}\right\}
\end{aligned}
$$


In (5.111) the constants $c_{1}, i=1,2$ are chosen to have masses $\rho_{1}$ (respectively $\rho_{2}$ ) for the steady states. Note that here the size of the tail of $g_{i, \infty}(v)$ is proportional to $\gamma_{i}$. Hence, the smaller the $\gamma$ is, the smaller the number of bounded moments of the steady state is. Taking the sum of the densities in (5.111) gives the expression of the total density

$$
g_{\infty}(v)=\frac{1}{\rho}\left(\frac{c_{1}}{v^{2+2 \gamma_{1} / \lambda}}+\frac{c_{2}}{v^{2+2 \gamma_{2} / \lambda}}\right) \exp \left\{-\frac{2\left(\gamma_{1} m_{1}+\gamma_{2} m_{2}\right)}{\lambda \rho v}\right\} .
$$

The analysis of the steady density (5.112) reveals that, depending of the values of the various parameters involved, one can have the formation of a bimodal distribution. In fact, the extremals of the (nonnegative) function

$$
\psi(v)=\left(\frac{a}{v^{p}}+\frac{b}{v^{q}}\right) \exp \{-c / v\}
$$

where $a, b, c$ are positive constants, and $q>p$, are located in the points solutions of the equation

$$
\Phi(v)=-p a v^{q-p+1}-q b v+c a v^{q-p}+c b=0 .
$$

On the other hand, since $\Phi(0)>0$, while $\Phi(+\infty)=-\infty$, the curve $y=\Phi(v)$ crosses the axis $y=0$ either in a single point or in three points. In this last case, we have two maxima and one minimum outside $v=0$, and consequently a bimodal distribution.

\section{Two-dimensional models}

The Chatterjee-Chakrabarti-Manna (CCM) model introduces into Angle's original trade a noticeable novelty. Arguing that agents are not indistinguishable in reality, but have personal trading preferences, Chatterjee et al. [21] introduced the concept of quenched saving propensity. Now $\lambda$ is not a global quantity, but characterizes the agents. The current "state" of an agent is consequently described by two numbers, his wealth $w>0$ and his personal saving propensity $\lambda \in(0,1)$. We shall only discuss the case where $\lambda$ does not change with time. Trade rules which allow the agents to adapt their saving strategy in time ("annealed saving") have been investigated [21, 19], but seemingly do not exhibit genuinely novel effects.

\subsection{Saving propensity as additional variable}

The configuration of the kinetic system is described by the extended density function $f(t ; \lambda, w)$. The wealth distribution $h(t ; w)$ is recovered from the density $f(t ; \lambda, w)$ as marginal,

$$
h(t ; w)=\int_{0}^{1} f(t ; \lambda, w) d \lambda
$$


but is no longer sufficient to characterize the configuration completely. The other marginal yields the time-independent density of saving propensities,

$$
\chi(\lambda)=\int_{\mathbb{R}_{+}} f(t ; \lambda, w) d w .
$$

Clearly, $\chi(\lambda)$ is determined by the initial condition $f(0 ; \lambda, w)$, and should be considered as defining parameter of the model. The collision rules are the same as originally (3.33), but take into account the individual characteristics: two agents with pre-trade wealth $v, w$ and saving propensities $\lambda, \mu$, respectively, exchange wealth according to

$$
\begin{aligned}
v^{*} & =\lambda v+\epsilon[(1-\lambda) v+(1-\mu) w], \\
w^{*} & =\mu w+(1-\epsilon)[(1-\lambda) v+(1-\mu) w] .
\end{aligned}
$$

Clearly, wealth is strictly conserved, $v^{*}+w^{*}=v+w$, so the mean wealth $M$ is constant in time. The Boltzmann equation (2.18) is now posed on a twodimensional domain, $(\lambda, w) \in(0,1) \times(0, \infty)$. The collisional gain operator $Q_{+}$ satisfies

$$
Q_{+}(f, f)[\varphi](\lambda)=\int_{\mathbb{R}_{+}^{2}} \int_{0}^{1}\left\langle\varphi\left(v^{*}\right)\right\rangle f(\lambda, v) f(\mu, w) d v d w d \mu
$$

after integration against a regular test function $\varphi(w)$. For simplicity, we assume that $\epsilon$ is symmetric around $1 / 2$.

\subsubsection{Pareto tail of the wealth distribution}

Due to its two-dimensionality, the CCM model behaves very different from the strictly conservative model (3.33). In particular, $h_{\infty}(w)$ may possess a Pareto tail. In analogy to $\mathbf{S}(s)$ from (3.22), define the function

$$
\mathbf{Q}(r):=\int_{0}^{1} \frac{\chi(\lambda)}{(1-\lambda)^{r}} d \lambda,
$$

which determines the properties of the steady wealth distribution $h_{\infty}(w)$ as follows [47]:

(PT') if $\mathbf{Q}(1)<+\infty$, and $\alpha \in[1,+\infty)$ is the infimum of $r$ for which $\mathbf{Q}(r)=+\infty$, then $h_{\infty}(w)$ has a Pareto tail of index $\alpha$;

(ST') if $\mathbf{Q}(r)<+\infty$ for all $r \geq 1$, then $h_{\infty}(w)$ has a slim tail;

(DD') if $\mathbf{Q}(1)=+\infty$, then $h_{\infty}(w)=\delta_{0}(w)$.

To derive these results, it is useful to think of the global wealth distribution $h_{\infty}(w)$ as superposition of $\lambda$-specific steady wealth distributions $f_{\infty}(\lambda, w) / \chi(\lambda)$, i.e., the wealth distributions of all agents with a certain personal saving propensity $\lambda$. The individual $\lambda$-specific distributions are conjectured $[21,52]$ to resemble the wealth distributions associated to the one-dimensional model (3.33), but 
their features are so far unknown. However, they are conveniently analyzed in terms of the $\lambda$-specific moments

$$
\hat{M}_{s}^{*}(\lambda)=\frac{1}{\chi(\lambda)} \int_{\mathbb{R}_{+}} w^{s} f_{\infty}(\lambda, w) d w
$$

Integration of the stationary Boltzmann equation

$$
f_{\infty}(\lambda, w)=Q_{+}\left(f_{\infty}, f_{\infty}\right)
$$

against $\varphi(w)=w^{s}$ for a non-negative integer $s$ gives

$\hat{M}_{s}^{*}(\lambda)=\frac{1}{\chi(\lambda)} \int_{\mathbb{R}_{+}^{2}} \int_{0}^{1}\left\langle([\lambda+\epsilon(1-\lambda)] v+\epsilon(1-\mu) w)^{s}\right\rangle f_{\infty}(\lambda, v) f_{\infty}(\mu, w) d \mu d v d w$

After simplifications,

$$
\begin{aligned}
& (1-\lambda) \phi_{s}(\lambda) \hat{M}_{s}^{*}(\lambda) \\
& \quad=\sum_{k=0}^{s-1}\left(\begin{array}{l}
s \\
k
\end{array}\right)\left\langle\epsilon^{s-k}[\lambda+\epsilon(1-\lambda)]^{k}\right\rangle \hat{M}_{s}^{*}(\lambda) \int_{0}^{1}(1-\mu)^{s-k} \hat{M}_{s-k}^{*}(\mu) \chi(\mu) d \mu,
\end{aligned}
$$

where $\phi_{s}(\lambda)$ is a polynomial with no roots in $[0,1]$. The $\lambda$-specific steady wealth distributions have slim tails, and moments of arbitrary order can be calculated recursively from (6.123). From

$$
\hat{M}_{0}^{*}(\lambda) \equiv 1, \quad \hat{M}_{1}^{*}(\lambda)=\frac{M}{\mathbf{Q}(1)}(1-\lambda)^{-1}
$$

it follows inductively that

$$
\hat{M}_{s}^{*}(\lambda)=r_{s}(\lambda)(1-\lambda)^{-s}
$$

and $r_{s}(\lambda)$ is a continuous, strictly positive function for $0 \leq \lambda \leq 1$. By Jensen's inequality, formula (6.125) extends from integers $s$ to all real numbers $s \geq 1$. In conclusion, the total momentum

$$
M_{s}^{*}=\int_{0}^{1} \hat{M}_{s}^{*}(\lambda) d \lambda \propto \int_{0}^{1} \frac{\chi(\lambda)}{(1-\lambda)^{s}} d \lambda
$$

is finite exactly if $\mathbf{Q}(s)$ is finite.

$\mathbf{Q}(1)=+\infty$ would imply infinite average wealth per agent in the steady wealth distribution by formula (6.126). This clearly contradicts the conservation of the mean wealth at finite times. In reality, the first moment vanishes, and $h_{\infty}$ is a Dirac distribution; see Section 6.1.3.

We emphasize this fact since a noticeable number of theoretical and numerical studies has been devoted to the calculation of $h_{\infty}$ for uniformly distributed 
$\lambda$, i.e. $\chi(\lambda) \equiv 1$, where clearly $\mathbf{Q}(1)=+\infty$. In the corresponding experiments $[21,22,52,19,25]$ with finite ensembles of $N$ agents, an almost perfect Pareto tail $h_{\infty}(w)=C_{N} w^{-2}$ of index $\alpha=1$ has been observed over a wide range $w_{N}<w<W_{N}$. However, the "true" tail of $h_{\infty}(w)-$ for $w \gg W_{N}-$ is slim. As the systems size $N$ increases, also $W_{N} \propto N$ increases and $C_{N} \propto 1 / \ln N \rightarrow 0$. In fact, one proves [47] weak convergence of $h_{\infty}(w)$ to $\delta_{0}(w)$ in the thermodynamic limit $N \rightarrow \infty$.

\subsubsection{Rates of relaxation: Pareto tail}

The discussion of relaxation is more involved than in one dimension, and we restrict our attention to the deterministic CCM model, $\epsilon \equiv 1 / 2$, in the case (PT') of Pareto tails of index $\alpha>1$. In fact, it is believed [22] that the randomness introduced by $\epsilon$ has little effect on the large-time behavior of the kinetic system.

The stationary state of the deterministic CCM model is characterized by the complete stop of wealth exchange. This is very different from the steady states for the one-dimensional models, where the macroscopic wealth distribution is stationary despite the fact that wealth is exchanged on the microscopic level. Stationarity in (6.117) and (6.118) is achieved precisely if $v(1-\lambda)=w(1-\mu)$ for arbitrary agents with wealth $v, w$ and saving propensities $\lambda, \mu$, respectively. Correspondingly, the particle density concentrates in the plane on the curve

$$
K_{\infty}=\{(\lambda, w) \mid(1-\lambda) w=M / \mathbf{Q}(1)\},
$$

and the steady wealth distribution is explicitly given by Mohanty's formula [48],

$$
h_{\infty}(w)=\frac{M}{w^{2}} \chi\left(1-\frac{M}{w}\right)
$$

with the convention that $\chi(\lambda)=0$ for $\lambda<0$.

The conjectured $[25,20]$ time scale for relaxation of solutions is $t^{-(\alpha-1)}$,

$$
\lim _{t \rightarrow \infty} \frac{\ln \mathbf{W}\left[h(t ; w), h_{\infty}(w)\right]}{-\ln t}=\alpha-1 .
$$

It has been proven [34] for all $\alpha>1$ that the limit in (6.129) is at most $\alpha-1$, i.e. relaxation cannot occur on a faster time scale. The complete statement (6.129), however, was made rigorous only for $1<\alpha<2$ so far [47].

The key tool for the analysis is the equation for the $\lambda$-specific mean wealth,

$$
\frac{d}{d t} \hat{M}_{1}(t ; \lambda)=-\frac{1-\lambda}{2} \hat{M}_{1}(t ; \lambda)+\int_{0}^{1} \frac{1-\mu}{2} \hat{M}_{1}(t ; \mu) \chi(\mu) d \mu
$$

Intuitively, the slow algebraic relaxation is explained by the temporal behavior of the richest agents. By (6.130), the $\lambda$-specific average wealth $\hat{M}_{1}(t ; \lambda)$ grows at most linearly in time,

$$
\hat{M}_{1}(t ; \lambda) \leq t+\hat{M}_{1}(0 ; \lambda) .
$$


Thus, the tail of the wealth curve $h(t ; w)$ becomes slim for $w \gg t$. The cost of transportation in (2.8) to "fill up" the fat tail $h_{\infty}(w) \propto w^{-(\alpha+1)}$ is approximately given by

$$
\int_{t}^{\infty} w h_{\infty}(w) d w \propto \int_{t}^{\infty} w^{-\alpha} d w \propto t^{-(\alpha-1)} .
$$

That equilibration works no slower than this (at least for $1<\alpha<2$ ) follows from a detailed analysis of the relaxation process. In [47], it has been proven that

$$
\int_{0}^{1}\left|\hat{M}_{1}(t ; \lambda)-\frac{M}{\lambda \mathbf{Q}(1)}\right| \chi(\lambda) d \lambda \propto t^{-(\alpha-1)}
$$

by relating (6.130) to the radiative transfer equation [39]. Moreover, the $\lambda$ specific variance

$$
\hat{V}(t ; \lambda)=\hat{M}_{2}(t ; \lambda)-\hat{M}_{1}(t ; \lambda)^{2}
$$

was shown to satisfy

$$
\int_{0}^{1}(1-\lambda)^{2} \hat{V}(t ; \lambda) \chi(\lambda) d \lambda \propto t^{-\alpha}
$$

provided $1<\alpha<2$. Combination of (6.133) and (6.135) leads to (6.129).

Moreover, relaxation may be decomposed into two processes. The first is concentration of agents at the $\lambda$-specific mean wealth $\hat{M}_{1}(t ; \lambda)$; i.e., all agents with the same saving propensity become approximately equally rich. According to (6.135), this process happens on a time scale $t^{-\alpha / 2}$. Second, the localized mean values tend towards their respective terminal values $M / \lambda \mathbf{Q}(1)$. Thus, agents of the same saving propensity simultaneously "adjust" their wealth. By (6.133), the respective time scale is $t^{-(\alpha-1)}$, which is indeed slower than the first provided $\alpha<2$.

\subsubsection{Rates of relaxation: Dirac delta}

Finally, the deterministic CCM model is considered with a density $\chi(\lambda)$ where $\lim _{\lambda \backslash 0} \chi(\lambda)>0$, e.g. $\chi \equiv 1$ on $[0,1]$. Clearly, $\mathbf{Q}(1)=+\infty$. An analysis of (6.130) provides [47] for $\lambda<1$ the estimate

$$
\frac{c}{1-\lambda} \leq \ln t \cdot \hat{M}_{1}(t ; \lambda) \leq \frac{C}{1-\lambda} \quad\left(t>T_{\lambda}\right),
$$

with $0<c<C<+\infty$, and $T_{\lambda} \rightarrow+\infty$ as $\lambda \rightarrow 1$. Convergence of $h(t ; w)$ to a Delta in $w=0$ is a direct consequence, since $\hat{M}_{1}(t ; \lambda)$ tends to zero for each $0 \leq \lambda<1$ as $t \rightarrow \infty$.

Estimate (6.136) has a direct interpretation. Agents of very high saving propensity $\lambda \approx 1$ drain all wealth out of the remaining society as follows. At intermediate times $t \gg 1$, agents equilibrate in microscopic trades so that the product $(1-\lambda) w$ becomes approximately a global constant $m(t)$. Agents with low saving propensity $\lambda<1-m(t) / t$ indeed satisfy $w \approx m(t) /(1-\lambda)$. Agents 
with higher saving propensity, however, are in general far from this (apparent) equilibrium; their target wealth $m(t) /(1-\lambda)$ is very large, whereas their actual wealth is bounded by $t$ on the average. Correspondingly, a "Pareto region" of the shape $h(t ; w) \approx \chi(1) m(t) w^{-2}$ forms over a range $1 \ll w \leq t$, whereas the tail of $h(t ; w)$ for $w \gg t$ is slim. The average wealth per agent contained in the Pareto region amounts to

$$
\int_{1}^{t} w h(t ; w) d w \approx \chi(1) m(t) \ln t .
$$

By conservation of the average wealth, the global constant $m(t)$ tends to zero logarithmically in $t$ and gives rise to (6.136).

\subsection{A Fokker-Planck equation for distributed trading rate}

In the previous section we analyzed the two-dimensional model of ChatterjeeChakrabarti-Manna, namely a kinetic model of Boltzmann type, in which the saving propensity represents an additional variable. In the same spirit, a twodimensional model of Fokker-Planck type has been recently described in [37]. The idea is to generalize the Fokker-Planck system (5.105) to the case in which the trading rate is randomly distributed on the interval $(0,1)$, with distribution $\Gamma(\lambda)$, where

$$
\Gamma(\lambda)=\int_{\mathbb{R}_{+}} g_{0}(\lambda, v) d v, \quad 0<\lambda<1,
$$

is the $\lambda$-marginal of the initial density of wealth. In this case, the unknown densities $g_{i}(\tau ; v)(i=1, \ldots, n)$ are substituted by $g(\tau ; \lambda, v)$, while $\sum_{j=1}^{n} g_{i}$ becomes

$$
\langle g(\lambda, v)\rangle_{\lambda}=\int_{0}^{1} g(\lambda, v) d \lambda .
$$

Note that here and below we will denote

$$
\langle\Phi(\lambda)\rangle_{\lambda}=\int_{0}^{1} \Phi(\lambda) d \lambda .
$$

If we assume $\mu_{i j}=\mu$ and $\tau_{i j}=1(i, j=1, \ldots, n)$ in (5.105), the corresponding Fokker-Planck equation with a continuous varying trading rate reads

$$
\begin{aligned}
\frac{\partial g(\lambda, v)}{\partial \tau}=\frac{\mu}{2} \frac{\partial^{2}}{\partial v^{2}}\left(v^{2}\langle\rho(\tau ; \lambda)\rangle_{\lambda} g(\lambda, v)\right) & \\
& +\frac{\partial}{\partial v}\left(\left(\lambda v\langle\rho(\tau ; \lambda)\rangle_{\lambda}-\langle\lambda M(\tau ; \lambda)\rangle_{\lambda}\right) g(\lambda, v)\right) .
\end{aligned}
$$

Taking into account that the total mass is preserved, $\langle\rho(\lambda)\rangle_{\lambda} \equiv 1$, while the distribution

$$
\Gamma(\tau ; \lambda)=\int_{\mathbb{R}_{+}} g(\tau ; \lambda, v) d v
$$


does not depend on time, i.e. $\Gamma(\tau ; \lambda)=\Gamma(\lambda)$, equation (6.138) simplifies to

$$
\frac{\partial g(\lambda, v)}{\partial \tau}=\frac{\mu}{2} \frac{\partial^{2}}{\partial v^{2}}\left(v^{2} g(\lambda, v)\right)+\frac{\partial}{\partial v}\left(\left(\lambda v-\langle\lambda M(\lambda)\rangle_{\lambda}\right) g(\lambda, v)\right) .
$$

The analytical study of the behavior of the solution to equation (6.138) would certainly deserve attention. The main difference between the CCM model and the present one is related to the fact that the Fokker-Planck equation (6.139) is obtained from the CPT model with risky components. A detailed study of the Fokker-Planck equation (6.139) is forthcoming.

\section{Numerical experiments}

The numerical simulation of kinetic equations of Boltzmann type is in general a challenging problem, due both to the nonlinearity of the collision operator, and to the presence of fat tails in various dissipative systems. A recent overview of the main existing numerical methods can be seen by looking at the recent books $[31,49]$. The most widely used methods for the discretization of Boltzmann type collisional equations are Monte Carlo methods, spectral methods and finitedifference methods. To verify the analytical results for the relaxation behavior of the wealth kinetic models, we resort to Monte Carlo simulations. This approach is quite standard to reconstruct steady distributions, even if, in presence of fat tails for the large-time wealth curve, one has to deal additionally with the problem of reconstructing the profile in the zone in which there are few particles. On the other hand, it is not clear that a marked improvement can be obtained by resorting to spectral methods, where the large-velocity zone is discarded. This approximation produces a small error in classical kinetic theory, where the stationary Maxwellian distribution decays exponentially fast, but it could destroy completely the fat tailed part. Numerical experiments by means of Monte Carlo methods have been recently done in [34,35] for both the CPT and the CCM models. Kinetic system (5.95) has been subsequently investigated via analogous methods to show the (eventual) formation of a bimodal steady distribution [37]. In view of their interest in application, we present here the main results, together with a brief presentation of the underlying numerical method.

In these rather basic simulations, known as direct simulation Monte Carlo (DSMC) method or Bird's scheme, pairs of agents are randomly and nonexclusively selected for binary collisions, and exchange wealth according to the respective trade rules. One time step corresponds to $N / 2$ such interactions, with $N$ denoting the number of agents. In all experiments, every agent possesses unit wealth initially.

The state of the kinetic system at time $t>0$ is characterized by the $N$ wealth values $w_{1}(t), \ldots, w_{N}(t)$ in the CPT simulations, and additionally by the saving propensities $\lambda_{1}, \ldots, \lambda_{N}$ for CCM. The densities for the current wealth $f^{(N)}(t ; w)$ and the steady state $f_{\infty}^{(N)}$ are each a collection of scaled Dirac Deltas 
at positions $w_{i}$. The associated distribution functions are build of a sequence of rectangles,

$$
F^{(N)}(t ; w)=\#\left\{\text { agents with wealth } w_{i}(t)>w\right\} / N,
$$

and respectively for $F_{\infty}^{(N)}(w)$.

The goal is to monitor the convergence of the wealth distribution $f^{(N)}(t ; w)$ to the approximate steady state $f_{\infty}^{(N)}(w)$ over time in terms of the Wassersteinone-distance. This amounts to computing the area between the two distribution functions $F^{(N)}(t ; w)$ and $F_{\infty}^{(N)}(w)$, which is performed as follows. The starting point is represented by two arrays of length $N$, one containing the current wealth values $w_{i}(t)$, and one the steady state data $w_{i}(\infty)$. One concatenates these arrays, sorts them in ascending order, and computes the array of differences between consecutive elements. This array represents the widths of the rectangles. To construct the array of the rectangles' heights, one concatenates two arrays of length $N$ containing the entries $1 / N$ and $-1 / N$, respectively, into one, and permutes it in the same way as the wealth vector in the step before. The absolute value of this array's cumulative sum represents the heights. The Wassersteinone-distance is now readily obtained by evaluation of the scalar product of width and height vector.

\subsection{CPT model}

The relaxation behavior of the CPT model (3.35) is investigated when the random variables $\eta_{1}, \eta_{2}$ attain values $\pm \mu$ with probability $1 / 2$ each. According to the analytical results, the shape of the steady state can be determined from Figure 3.1. Results are reported for zones II and III. Recall that zone I is forbidden by the constraint $|\mu|<\lambda$, whereas parameters in zone IV lead to wealth condensation (without convergence in Wasserstein metrics). For zones II and III simulations are performed for systems consisting of $N=500, N=5000$ and $N=50000$ agents, respectively.

The relaxation in the CPT model occurs exponentially fast. Though the system has virtually reached equilibrium after less than $10^{2}$ time steps, simulations are performed for $10^{4}$ time steps. In order to obtain a smooth result, the wealth distribution is averaged over another $10^{3}$ time steps. The resulting reference state $P_{\infty}^{(N)}$ is used in place of the (unknown) steady wealth curve.

For zones II and III a risk index of $\mu=0.1$, and a saving propensity of $\lambda \equiv 0.7$ for zone II and $\lambda \equiv 0.95$ for zone III, respectively, are chosen. The nontrivial root of $\mathbf{S}(s)$ in (3.22) is $\bar{s} \approx 12.91$ in the latter case. For each choice of $N$ and each pair $(\mu, \lambda)$, averages over 100 simulations have been made. Figure 7.2 shows the decay of the Wasserstein-one-distance of the wealth distribution to the approximate steady state over time. In both zones, we observe exponential decay. The reason for the residual Wasserstein distance of order $10^{-2}$ lies in the statistical nature of this model, which never reaches equilibrium in finitesize systems, due to persistent thermal fluctuations. Note that before these 

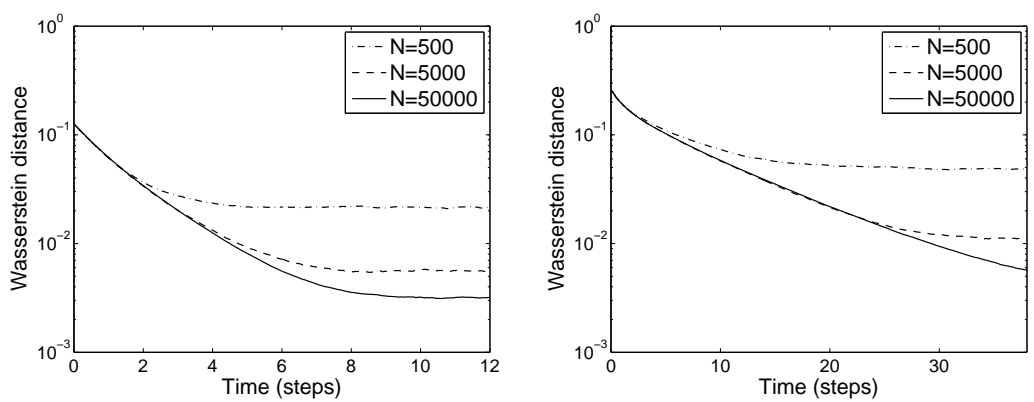

Figure 7.2: CPT model: Decay of the Wasserstein distance to the steady state in zones II (left) and III (right).

fluctuations become dominant, relaxation is extremely rapid. The exponential rate is independent of the number of agents $N$.

\subsection{CCM model}

The CCM model is expected to relax at an algebraic rate (6.129). As simulations indeed take much longer to reach equilibrium than in the case of CPT, the numerical experiments are carried out for about $10^{5}$ time steps, and then the wealth distribution is averaged over another $10^{4}$ time steps. Again, this reference state is used in place of the (unknown) steady wealth curve. The saving propensities for the agents are assigned at the beginning of each run and are kept fixed during this simulation. Agents are assigned the propensities $\lambda_{j}=1-\omega_{j}^{1 / 2.5}$, where the $\omega_{j} \in(0,1)$ are realizations of a uniformly distributed random variable. Simulations are performed for the deterministic situation $\epsilon \equiv 1 / 2$ as well as for uniformly distributed $\epsilon \in(0.4,0.6)$. In both situations, computations are carried out for systems consisting of $N=500, N=5000$ and $N=50000$ agents, respectively.

The steady state reached in one simulation is typically non-smooth, and smoothness is only achieved by averaging over different simulations. However, in contrast to the CPT model, the steady states for CCM do depend on the initial conditions, namely through the particular realization of the distribution of saving propensities $\lambda_{1}, \ldots, \lambda_{N}$ among the agents. Consequently, there are two possibilities to calculate the relaxation rates. One can monitor either the convergence of the wealth distributions in one run to the steady distribution corresponding to that specific realization of the saving propensities, or the convergence of the transient distributions, obtained from averaging over several simulations, to the single smooth steady state that results from averaging the simulation-specific steady states.

Figure 7.3 shows the evolution of the Wasserstein-one-distance of the wealth distributions to the individual steady states, both in the purely deterministic 

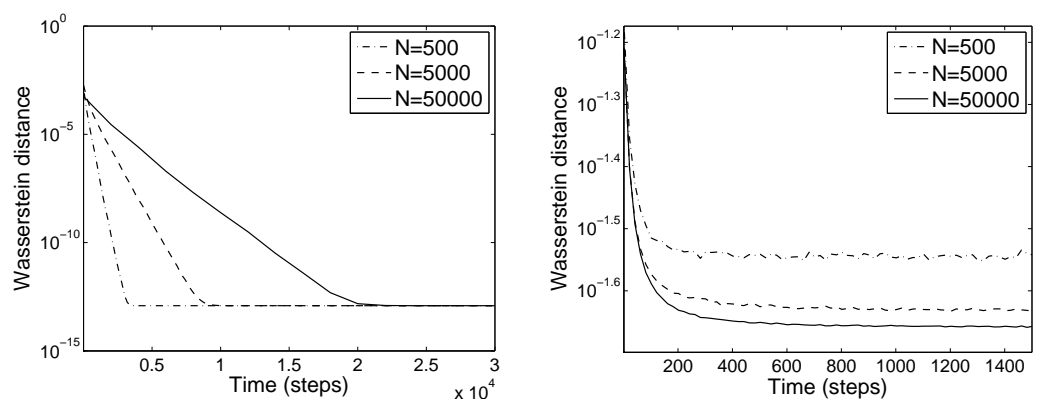

Figure 7.3: CCM model: Decay of the averaged Wasserstein distance to the steady states for $\varepsilon \equiv 1 / 2$ and for $\varepsilon \in(0.4,0.6)$ uniformly distributed.
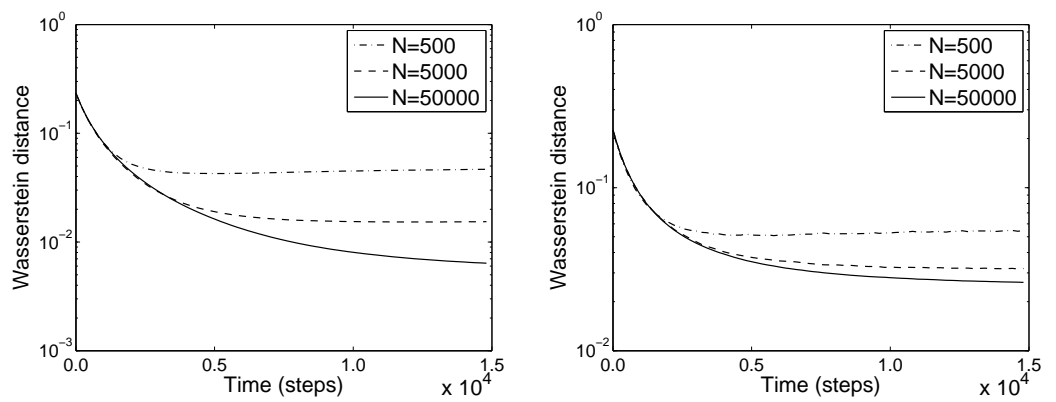

Figure 7.4: CCM model: Decay of the Wasserstein distance to the averaged steady state for $\varepsilon \equiv 1 / 2$ (left) and for $\varepsilon \in(0.4,0.6)$ uniformly distributed (right).

setting $\epsilon \equiv 1 / 2$ (left), and for uniformly distributed $\epsilon \in(0.4,0.6)$. (The curves in the figures represent averages of the Wasserstein distances calculated in the individual simulations.) In comparison, the distance of the simulation-averaged wealth distributions to the single (averaged) steady state is display in Figure 7.4. Again, results are shown for $\epsilon \equiv 1 / 2$ (left), and for uniformly distributed $\epsilon \epsilon$ $(0.4,0.6)$, respectively.

Some words are in order to explain the results. The almost perfect exponential (instead of algebraic) decay displayed in Figure 7.3 obviously originates from the finite size of the system. The exponential rates decrease as the system size $N$ increases. In the theoretical limit $N \rightarrow \infty$, one expects sub-exponential relaxation as predicted by the theory. We stress that, in contrast, the exponential decay rate for the CPT model in Figure 7.2 is independent of the system size. 

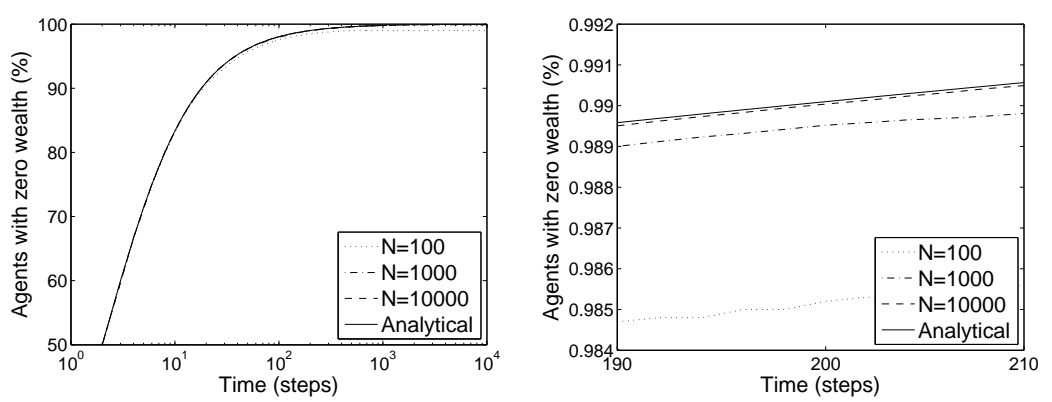

Figure 7.5: "Winner takes all" model: Evolution of the fraction of agents with zero wealth (left) and blow up (right).

\subsection{Winner takes all}

Third, the "Winner takes all" model (3.28) is simulated. As time evolves, all agents but one become pauper and give rise to a Dirac Delta at $w=0$. We run $M=100$ simulations for systems consisting of $N=100, N=1000$ and $N=10000$ agents, respectively. Figure 7.5 displays the simulation-averaged fraction of the population with zero wealth. This fraction of pauper agents grows linearly until a saturation effect becomes visible. The blow up figure shows the improving approximation of the theoretically predicted rate for growing system size.

\subsection{Bimodal distributions}

Finally, the Boltzmann system (5.95) has been investigated. The numerical experiments refer to the situation of two countries, i.e. $n=2$. It will be straightforward, however, to extend the following to the general situation of an arbitrary number of countries. Hence, let us consider

$$
\begin{aligned}
\frac{\partial}{\partial t} f_{1}(t ; w) & =\frac{1}{\tau_{11}} \mathcal{Q}\left(f_{1}, f_{1}\right)(w)+\frac{1}{\tau_{12}} \mathcal{Q}\left(f_{1}, f_{2}\right)(w) \\
\frac{\partial}{\partial t} f_{2}(t ; w) & =\frac{1}{\tau_{22}} \mathcal{Q}\left(f_{2}, f_{2}\right)(w)+\frac{1}{\tau_{21}} \mathcal{Q}\left(f_{2}, f_{1}\right)(w)
\end{aligned}
$$

Herein, $\mathcal{Q}\left(f_{1}, f_{1}\right)$ and $\mathcal{Q}\left(f_{2}, f_{2}\right)$ represent the collision operators which describe the change of density due to binary domestic trades, while $\mathcal{Q}\left(f_{1}, f_{2}\right), \mathcal{Q}\left(f_{2}, f_{1}\right)$ are the collision operators which describe the change of density due to binary international trades.

In previous simulations (cf. the examples of Sections 7.1, 7.2 and 7.3), pairs of agents are randomly and non-exclusively selected for binary collisions, and exchange wealth according to the trading rule under consideration. To extend this procedure to the present situation, we pursue the following approach. Let us indicate with $N_{i}(i=1,2)$ the number of traders of the two countries we will 

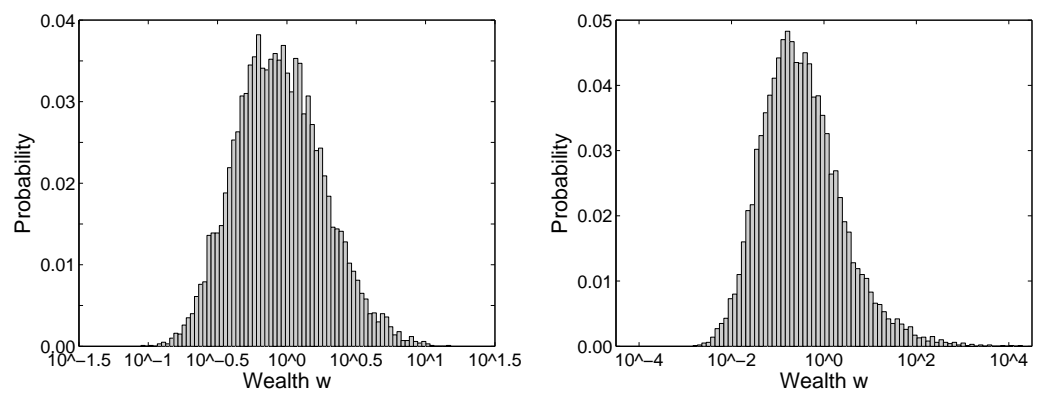

Figure 7.6: Histogram of steady state distribution for $\gamma_{1}=\gamma_{2}=0.875$ (left) and for $\gamma_{1}=\gamma_{2}=0.99$ (right).

take for our simulation. Assume without loss of generality, that $N_{1} \geq N_{2}$. One time step in our simulation corresponds to $N_{1}$ interactions. Since we have to perform trade events for both groups, each of these interactions has two stages, which are described in the following.

In the first stage, select randomly an agent from group 1 . Then select randomly a trading partner from the whole population, where the probabilities for each agent to be selected depend on $\tau_{11}, \tau_{12}$. For example, if the trading frequency in group 1 for domestic trades is twice as high as for international trades, the probability to select a trade partner from group 1 has to be twice the probability to select a trade partner from group 2 . Once the trade partner is selected, the trade takes place and wealth is exchanged according to the trading rule (5.93).

In the second stage, we need to perform trades for group 2. Since $N_{2} \leq$ $N_{1}$, we only perform a trade for group 2 in every $k$-th interaction, where $k=$ $\left\lceil N_{1} / N_{2}\right\rceil$. If a trade is carried out, it is done similarly as for group 1: select randomly an agent from group 2 and a partner from the whole population, where the probabilities for an agent to be selected as a partner depend on $\tau_{21}, \tau_{22}$. Then, carry out the trade according to the trading rule (5.93).

In all our experiments, every agent possesses unit wealth initially. The relaxation in the CPT model occurs exponentially fast [34]. Hence, to compute a good approximation of the steady state it suffices to carry out the simulation for about $10^{4}$ time steps, and then average the wealth distribution over another 1000 time steps. In every experiment, we average over $M=100$ such simulation runs.

In a first example, we consider two groups with $N_{1}=N_{2}=5000$ agents. We investigate the relaxation behavior when the random variables $\eta_{i j}, i, j \in\{1,2\}$, attain values $\pm \mu$ with probability $1 / 2$ each. We set the coefficient $\gamma=1$. We set $\mu=0.15$ and $\tau_{i j}=1$ for $i, j \in\{1,2\}$. If we choose $\gamma_{1}=\gamma_{2}=0.875$ and $\gamma_{1}=\gamma_{2}=0.99$, respectively, the system reduces to the standard CPT model. The probability density for both cases is plotted in Figure 7.6. The cumulative 

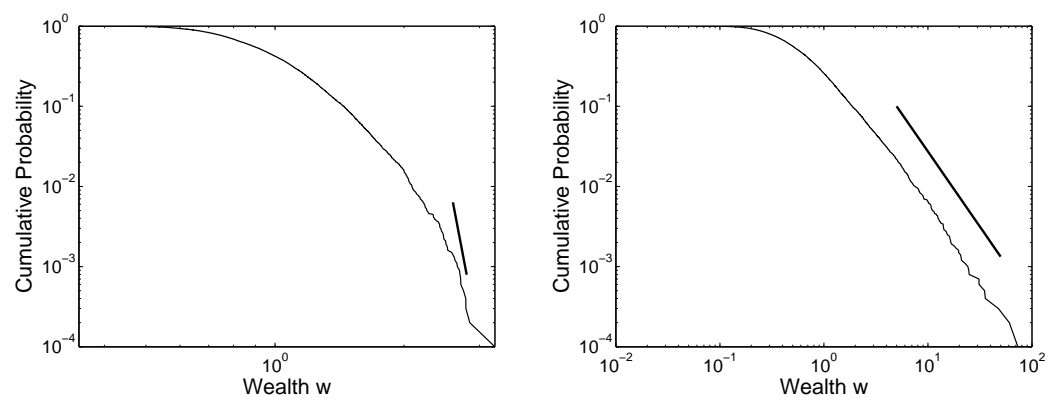

Figure 7.7: Cumulative wealth distribution for $\gamma_{1}=\gamma_{2}=0.875$ (left) and for $\gamma_{1}=\gamma_{2}=0.99$ (right).
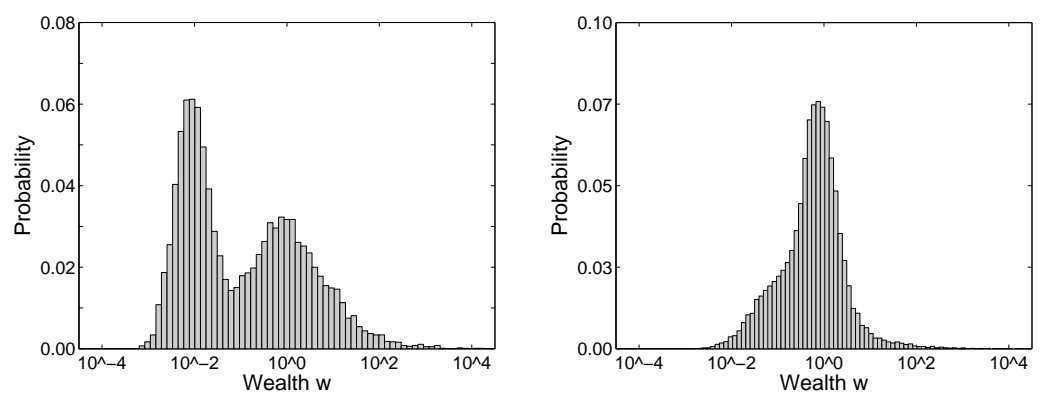

Figure 7.8: Histogram of steady state distribution for $\gamma_{1}=0.875$ and $\gamma_{2}=0.99$ (left) in comparison with the histogram for the union two disjunct populations with the same parameters (right).

distribution functions show a Pareto tail; see Figure 7.7. The Pareto index $\alpha$ of the tail is determined by the non-trivial root of (3.37) - strictly speaking this holds for the limit $N_{1,2} \rightarrow \infty-$, which is given by 28.068 and 1.875, respectively. These tail indices are indicated in Figure 7.7 by a thick line.

Now, we choose $\gamma_{1}=0.875$ and $\gamma_{2}=0.99$ and keep $\mu=0.15$ and $\tau_{i j}=1$ for $i, j \in 1,2$. The probability density for the whole population is plotted in Figure 7.8 (left plot). It shows a bimodal shape. The distance of the two peaks in the distribution decreases with decreasing difference between $\gamma_{1}$ and $\gamma_{2}$. Such bimodal distributions (and a polymodal distribution, in general) are also reported with real data for the income distributions in Argentina [38, 42]. This distribution features transport of wealth from one group to the other, which makes it different from the probability distribution for the union of two groups with the same parameters which do not interact, see Figure 7.8 (right plot).

The associated cumulative distribution functions are shown in Figure 7.9.

The cumulative distribution functions for the two groups are shown in Figure 

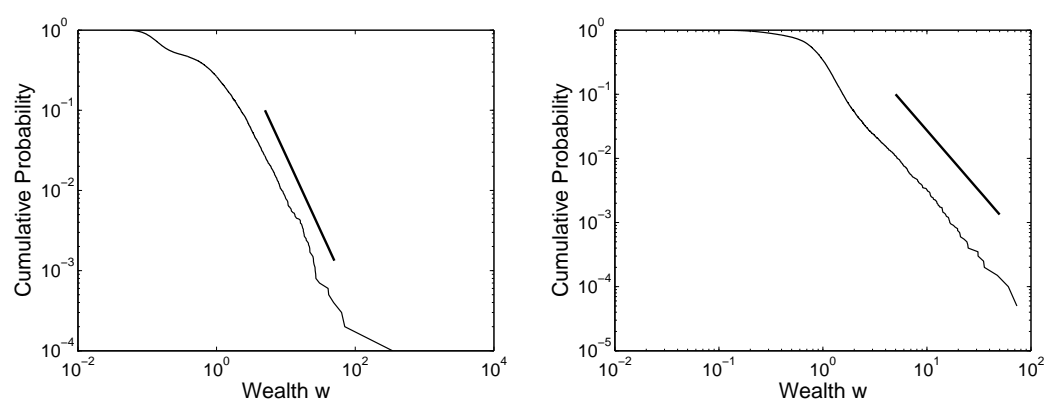

Figure 7.9: Cumulative wealth distribution for $\gamma_{1}=0.875$ and $\gamma_{2}=0.99$ (left) in comparison with the cumulative wealth distribution for the union two disjunct populations with the same parameters (right).
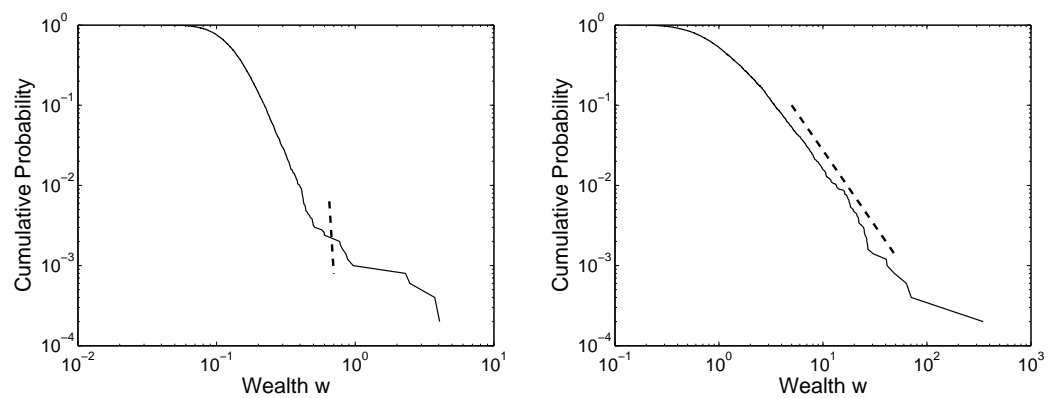

Figure 7.10: Cumulative wealth distribution for group 1 with $\gamma_{1}=0.875$ (left) and group 2 with $\gamma_{2}=0.99$ (right).

7.10. For comparison we plot the same Pareto tail index lines as in Figure 7.7.

\section{Conclusions}

We have reviewed and compared various approaches to model the dynamics of wealth distribution in simple market economies. The considered models were based on a kinetic description of the binary trade interactions between the agents, comparable to collisions between molecules in a homogeneous gas. The macroscopic statistics of the models display wealth distributions that are in agreement with empirical data.

The existing kinetic models can be mainly classified into two groups, depending if the total wealth is preserved into a single trade, or not. Conservative models, that belongs to the first class, can be further divided into two smaller groups, which are characterized by the property that the total wealth is pre- 
served pointwise, or in the mean. The risky market approach (CPT) by Cordier et al. [29] belongs to this last class, while the model with quenched saving propensities (CCM) by Chatterjee et al. [21] is obtained from pointwise conservative trades. Both models constitute refinements of the original idea developed by Angle [2]. For CPT, randomness - related to the unknown outcome of risky investments - plays the pivotal rôle. In contrast to Angle's original model, the market risk is defined in a way that breaks the strict conservation of wealth in microscopic trades and replaces it by conservation in the statistical mean. The founding idea of CCM is to incorporate individual trading preferences by assigning personal saving propensities to the agents. For suitable choices of the respective model parameters, both approaches are able to produce realistic Pareto tails in the wealth distribution. In direct comparison, the CPT model appears more natural, since the dependence of the stationary wealth distribution on the system parameters is more robust, and the steady state is exponentially attracting in contrast to algebraic relaxation for CCM.

Second, nonconservative trades model were considered. Here, the pioneering idea goes back to Slanina [54], who first noticed the robust connection between the increasing of the average wealth in a simple market society, and the cooling of a dissipative gas in classical kinetic theory. In this important group of models, tails are developed in consequence of the self-similar scaling. In particular, a Gamma distribution of Amoroso type [1] is produced through this approach in a simple market society in which the mean wealth collapses (the cooling phenomenon in dissipative kinetic theory!). The mathematical description takes advantage from the analogous methods introduced to describe dissipative Maxwell gases $[8,16]$. The methods allow to recover precise analytical details for the description of the self-similar profiles.

An important finding is that one must be careful with numerical simulations when delicate features like Pareto tails are concerned. The simulated ensembles in kinetic Monte Carlo experiments are necessarily of finite size, and the qualitative features of finite-size systems differ in essential points from those proven for the continuous limit. Most remarkably, the finite-size CCM model exhibits non-trivial steady states with (apparent) Pareto tail in situations where the continuous model produces a Dirac distribution. Also, the typical time scale for relaxation in the deterministic CCM model changes from exponential convergence (finite size) to algebraic convergence (continuous).

It is arguable which kind of approach (finite size or continuous) provides the better approximation to reality. However, it is important to notice that the predictions are qualitatively different. This should be kept in mind in the further development of these (currently over-simplistic) models.

\section{Acknowledgements}

The authors acknowledge support from the Italian MIUR, project "Kinetic and hydrodynamic equations of complex collisional systems". Bertram Düring is supported by the Deutsche Forschungsgemeinschaft, grant JU 359/6 (Forscher- 
gruppe 518). Daniel Matthes is supported by the Deutsche Forschungsgemeinschaft, grant JU 359/7. Bertram Düring and Daniel Matthes thank the Department of Mathematics of the University of Pavia, where a part of this research has been carried out, for the kind hospitality.

\section{References}

[1] L. Amoroso, Ricerche intorno alla curva dei redditi. Ann. Mat. Pura Appl. Ser. 421 (1925), 123-159.

[2] J. Angle, The surplus theory of social stratification and the size distribution of personal wealth. Social Forces 65(2) (1986), 293-326.

[3] L. Baringhaus and R. Grübel, On a class of characterization problems for random convex combination. Ann. Inst. Statist. Math. 49(3) (1997), 555567.

[4] A. Baldassarri, U. Marini Bettolo Marconi and A. Puglisi, Kinetic models of inelastic gases. Mat. Mod. Meth. Appl. Sci. 12 (2002), 965-983.

[5] D. Ben-Avraham, E. Ben-Naim, K. Lindenberg and A. Rosas Self-similarity in random collision processes. Phys. Rev. E 68 (2003), R050103.

[6] E. Ben-Naim and P. Krapivski, Multiscaling in inelastic collisions. Phys. Rev. E 61 (2000), R5-R8.

[7] E. Ben-Naim and P. Krapivski, Nontrivial velocity distributions in inelastic gases. J. Phys. A 35 (2002), L147-L152.

[8] M. Bisi, J.A. Carrillo and G. Toscani, Decay rates in probability metrics towards homogeneous cooling states for the inelastic Maxwell model. $J$. Stat. Phys. 124 (2-4) (2006) 625-653.

[9] A.V. Bobylev, The method of the Fourier transform in the theory of the Boltzmann equation for Maxwell molecules. Dokl. Akad. Nauk SSSR 225 (1975), 1041-1044.

[10] A.V. Bobylev, The theory of the nonlinear spatially uniform Boltzmann equation for Maxwellian molecules. Sov. Sci. Rev. c 7 (1988), 111-233.

[11] A.V. Bobylev, J.A. Carrillo and I. Gamba, On some properties of kinetic and hydrodynamic equations for inelastic interactions. J. Stat. Phys. 98 (2000), 743-773; Erratum on: J. Stat. Phys. 103, (2001), 1137-1138.

[12] A.V. Bobylev and C. Cercignani, Self-similar asymptotics for the Boltzmann equation with inelastic and elastic interactions. J. Stat. Phys. 110 (2003), 333-375. 
[13] A.V. Bobylev and I.M. Gamba, Boltzmann equations for mixtures of Maxwell gases: exact solutions and power like tails. J. Stat. Phys. 124 (2006), 497-516.

[14] J.-F. Bouchaud and M. Mézard, Wealth condensation in a simple model of economy. Physica A 282 (2000), 536-545.

[15] Z. Burda, D. Johnston, J. Jurkiewicz, M. Kamiński, M.A. Nowak, G. Papp and I. Zahed, Wealth condensation ia pareto macroeconomies. Phys. Rev. E 65 (2002), 026102.

[16] J.A. Carrillo and G. Toscani, Contractive probability metrics ans asymptotic behavior of dissipative kinetic equations. Riv. Mat. Univ. Parma 6(7), (2007) 75-198.

[17] A. Chakraborti and B.K. Chakrabarti, Statistical mechanics of money: how saving propensity affects its distributions. Eur. Phys. J. B. 17 (2000), 167170 .

[18] B.K. Chakrabarti, A. Chakraborti, and A. Chatterjee, Econophysics and Sociophysics: Trends and Perspectives, Wiley VCH, Berlin, 2006.

[19] A. Chatterjee and B.K. Chakrabarti, Ideal-gas-like market models with savings: Quenched and annealed cases. Physica A 382 (2007), 36-41.

[20] A. Chatterjee and B.K. Chakrabarti, Kinetic Exchange Models for Income and Wealth Distributions. Eur. Phys. J. B 60 (2007), 135-149.

[21] A. Chatterjee, B.K. Chakrabarti, and S.S. Manna, Pareto law in a kinetic model of market with random saving propensity. Physica A 335 (2004), $155-163$.

[22] A. Chatterjee, B.K. Chakrabarti, and R.B. Stinchcombe, Master equation for a kinetic model of trading market and its analytic solution. Phys. Rev. E 72 (2005), 026126.

[23] A. Chatterjee, Y. Sudhakar, and B.K. Chakrabarti, Econophysics of Wealth Distributions New Economic Windows Series, Springer, Milan, 2005.

[24] A. Chakraborti, Distribution of money in model markets of economy. Int. J. Mod. Phys. C 13(10) (2002), 1315-1321 .

[25] M. Patriarca, A. Chakraborti, E. Heinsalu, and G. Germano, Relaxation in statistical many-agent economy models. Europ. Phys. J. B 57 (2007), $219-224$.

[26] M. Patriarca, A. Chakraborti, and K. Kaski, A statistical model with a standard Gamma distribution. Phys. Rev. E 70 (2004), 016104.

[27] R. Coelho, P. Richmond, J. Barry and S. Hutzler, Double power law in income and wealth distributions. Physica A 387 (2008), 3847-3851. 
[28] S. Cordier, L. Pareschi and C. Piatecki, Mesoscopic modelling of financial markets. J. Stat. Phys. (in press)

[29] S. Cordier, L. Pareschi and G. Toscani, On a kinetic model for a simple market economy. J. Stat. Phys. 120 (2005), 253-277.

[30] R. D'Addario, Intorno alla curva dei redditi di Amoroso. Riv. Italiana Statist. Econ. Finanza, 4(1) (1932).

[31] P. Degond, L. Pareschi and G. Russo, Modeling and Computational Methods for Kinetic Equations, Modeling and Simulation in Science, Engineering and Technology Series, Birkhäuser, Boston, 2004.

[32] L. Desvillettes, G. Furioli and E. Terraneo, Propagation of Gevrey regularity for solutions of Boltzmann equation for Maxwellian molecules. Trans. Amer. Math. Soc. (in press).

[33] A. Drăgulescu and V.M. Yakovenko, Statistical mechanics of money. Eur. Phys. Jour. B 17 (2000), 723-729.

[34] B. Düring, D. Matthes and G. Toscani, Exponential and algebraic relaxation in kinetic models for wealth distribution. In: "WASCOM 2007" Proceedings of the 14th Conference on Waves and Stability in Continuous Media, N. Manganaro et al. (eds.), pp. 228-238, World Sci. Publ., Hackensack, NJ, 2008.

[35] B. Düring, D. Matthes and G. Toscani, Kinetic Equations modelling Wealth Redistribution: A comparison of Approaches. Phys. Rev. E (in press).

[36] B. Düring and G. Toscani, Hydrodynamics from kinetic models of conservative economies. Physica A 384 (2007), 493-506.

[37] B. Düring and G. Toscani, International and domestic trading and wealth distribution. Commun. Math. Sci. (in press).

[38] J.C. Ferrero, The monomodal, polymodal, equilibrium and nonequilibrium distribution of money. In: Econophysics of Wealth Distributions, A. Chatterjee, S. Yarlagadda and B.K. Chakrabarti (eds.), Springer (Italy), 2005.

[39] E. Gabetta, P.A. Markowich and A. Unterreiter, A note on the entropy production of the radiative transfer equation. Appl. Math. Lett. 12(4) (1999), $111-116$.

[40] E. Gabetta, G. Toscani and B. Wennberg, The Tanaka functional and exponential convergence for non-cut-off molecules. Proceedings of the Second International Workshop on Nonlinear Kinetic Theories and Mathematical Aspects of Hyperbolic Systems (Sanremo, 1994). Transport Theory Stat. Phys. 25 no. 3-5 (1996), 543-554. 
[41] U. Garibaldi, E. Scalas and P. Viarengo, Statistical equilibrium in simple exchange games II. The redistribution game. Eur. Phys. Jour. B 60(2) (2007), 241-246.

[42] K. Gupta, Money exchange model and a general outlook. Physica A 359 (2006), 634-640.

[43] B. Hayes, Follow the money. American Scientist 90(5) (2002), 400-405.

[44] S. Ispolatov, P.L. Krapivsky and S. Redner, Wealth distributions in asset exchange models. Eur. Phys. Jour. B 2 (1998), 267-276.

[45] B. Mandelbrot, The Pareto-Lévy law and the distribution of income. International Economic Review 1 (1960), 79-106.

[46] D. Matthes and G. Toscani, On steady distributions of kinetic models of conservative economies. J. Stat. Phys. 130 (2008), 1087-1117.

[47] D. Matthes and G. Toscani, Analysis of a model for wealth redistribution. Kinetic Rel. Mod. 1 (2008), 1-22.

[48] P.K. Mohanty, Generic features of the wealth distribution in an ideal-gaslike market. Phys. Rev. E 74(1) (2006), 011117.

[49] L. Pareschi, G. Russo and G. Toscani, Modelling and Numerics of Kinetic Dissipative Systems, Nova Science Publ., New York, 2006.

[50] L. Pareschi and G. Toscani, Self-similarity and power-like tails in nonconservative kinetic models. J. Stat. Phys. 124(2-4) (2006), 747-779.

[51] V. Pareto, Cours d'Économie Politique, Lausanne and Paris, 1897.

[52] M. Patriarca, A. Chakraborti and G. Germano, Influence of saving propensity on the power-law tail of the wealth distribution. Physica A 369 (2006), 723-736.

[53] P. Repetowicz, S. Hutzler and P. Richmond, Dynamics of money and income distributions. Physica A 356 (2005), 641-654.

[54] F. Slanina, Inelastically scattering particles and wealth distribution in an open economy. Phys. Rev. E 69 (2004), 046102.

[55] H. Takayasu, Application of Econophysics, Springer, Tokyo, 2004.

[56] H. Takayasu, Practical fruits of econophysics, Springer, Tokyo, 2005.

[57] C. Villani, Topics in Optimal Transportation, Graduate Studies in Mathematics 58, American Mathematical Society, Providence, 2003.

[58] Y. Wang, N. Ding and L. Zhang, The circulation of money and holding time distribution. Physica A 324(3) (2003), 665-677. 
[59] V.M. Yakovenko, Statistical Mechanics Approach to Econophysics. In: Encyclopedia of Complexity and System Science, R.A. Meyers (ed.), Springer, New York, in press. 